\title{
Article \\ Robust Finite-Time Control Algorithm Based on Dynamic Sliding Mode for Satellite Attitude Maneuver
}

\author{
You $\mathrm{Li}^{1}{ }^{1}$ and Haizhao Liang ${ }^{2, *}$ \\ 1 School of Aerospace Science and Technology, Xidian University, Xi'an 710126, China; liyouhahaha@163.com \\ 2 School of Aeronautics and Astronautics, Sun Yat-sen University, Guangzhou 510275, China \\ * Correspondence: lianghch5@mail.sysu.edu.cn
}

check for

updates

Citation: Li, Y.; Liang, H. Robust Finite-Time Control Algorithm Based on Dynamic Sliding Mode for Satellite Attitude Maneuver. Mathematics 2022, 10, 111. https:// doi.org/10.3390/math10010111

Academic Editor: António M. Lopes

Received: 13 November 2021

Accepted: 24 December 2021

Published: 30 December 2021

Publisher's Note: MDPI stays neutral with regard to jurisdictional claims in published maps and institutional affiliations.

Copyright: (C) 2021 by the authors. Licensee MDPI, Basel, Switzerland. This article is an open access article distributed under the terms and conditions of the Creative Commons Attribution (CC BY) license (https:// creativecommons.org/licenses/by/ $4.0 /)$.

\begin{abstract}
Robust finite-time control algorithms for satellite attitude maneuvers are proposed in this paper. The standard sliding mode is modified, hence the inherent robustness could be maintained, and this fixed sliding mode is modified to dynamic, therefore the finite-time stability could be achieved. First, the finite -time sliding mode based on attitude quaternion is proposed and the loose finite-time stability is achieved by enlarging the sliding mode parameter. In order to get the strict finite-time stability, a sliding mode based on the Euler axis is then given. The fixed norm property of the Euler axis is used, and a sliding mode parameter without singularity issue is achieved. System performance near the equilibrium point is largely improved by the proposed sliding modes. The singularity issue of finite-time control is solved by the property of rotation around a fixed axis. System finite-time stability and robustness are analyzed by the Lyapunov method. The superiority of proposed controllers and system robustness to some typical perturbations such as disturbance torque, model uncertainty and actuator error are demonstrated by simulation results.
\end{abstract}

Keywords: finite-time control; robust control; dynamic sliding mode; satellite attitude maneuver

\section{Introduction}

With regard to the matter of satellite attitude control, the standard sliding mode is a mature and widely used control algorithm. The structure of the standard sliding mode for satellite attitude control is simple and has definite physical meaning. Moreover, the physical meaning brings strong robustness to some typical perturbations such as unknown disturbance, inertia matrix uncertainty and control actuator error. Some work [1-3] has been done on the design of sliding mode controllers and has demonstrated the superiority of the standard sliding mode. However, the system on this sliding mode has an exponential convergence rate, which means that with infinite time, the system could reach its equilibrium point strictly. However, some current space missions such as push-broom imaging and staring imaging demand the fast attitude maneuver capability. In order to improve system convergence rates, some researches focus on the field fast attitude maneuver. $\mathrm{Li}$, Ye and so on [4-7] have done some work to improve the convergence rate of standard controllers. They pointed out that the key to improve the system convergence rate is to design angular velocity properly. The maneuver stage with constant angular velocity is designed and the convergence rate could be maintained during the stage. However, most of the work did not solve the exponential convergence issue, and the terminal convergence rate could still be improved. Zhang [8], Verbin [9] and Rojsiraphisal [10] designed the "braking curve" of angular velocity for satellite attitude control. The trajectory of angular velocity is optimized and the trajectory of the slowing down process is designed. The focus of their work was improving the system convergence rate, but the exponential convergence rate issue still exists.

In order to get finite-time stability near the system equilibrium point, researchers have done a lot of work. Ye and Xiao and so on [11,12] designed finite-time controllers 
for satellite control. The focus of their work is the control torque allocation algorithm and the fault tolerant algorithm. Wu $[13,14]$ presented some methods to analyze finite time stability, such as the Lyapunov method and the terminal sliding mode method. The focus of his work is the structure of finite-time controllers for classic nonlinear systems and the standard structure of the terminal sliding mode. Liang, Wang and so on [15-17] designed finite-time controllers for satellite attitude control and the finite-time stability is analyzed by the Lyapunov method. Some typical Lyapunov functions are proposed in their works. Nguyen [18] designed a robust finite-time guidance law for maneuverable targets with unpredictable evasive strategies. Khelil [19] proposed a fast finite-time convergent guidance law with a nonlinear disturbance observer for unmanned aerial vehicle collision avoidance. Guo [20] designed a new continuous adaptive finite time guidance law against highly maneuvering targets. Generally, in order to get finite-time stability, the design of the controller needs some special modifications and the system loses the inherent strong robustness to perturbations. The design of robust finite-time controllers is another major concern of current research.

As discussed above, model uncertainty, unknown disturbance and actuator error are some typical perturbations in the satellite attitude system. In order to deal with perturbation issues, researchers have done some work. Xiao [21-23] designed fault tolerant controllers, and system model uncertainty and actuator error are estimated by the fault diagnosis function. However, the method is suitable for several typical uncertainty models but not suitable for the random noise model. Hu [24-26] designed some robust controllers to system uncertainty. The sign function terms are added in the controllers and the system uncertainty is treated as Gauss white noise with an upper bounded norm. However, the sign function terms would bring high frequency vibrations, which is harmful to the actuator and physical system. In order to deal with the uncertainty issue without bringing high frequency vibration, adaptive control was developed by some researchers. Qiao [27] and Gui [28] designed finite-tine attitude maneuver controllers considering the disturbance torque with Gauss white noise character and sine function character. Wang [29] and Ai [30] designed finite-time sliding modes for satellite attitude control, and the convergence time is estimated by the proposed methods and disturbance torque with consideration of the upper bounded norm. Some researchers [31-34] also designed finite time controllers for robot manipulator and vehicle systems. Generally, the finite-time controller considering overall perturbations still needs developing, and in order to deal with perturbations, the structure of finite-time controllers is relatively complex.

In this paper, the standard sliding mode will be modified to achieve finite-time stability. The strong robustness could be maintained by the similar structure with standard sliding mode and robust controllers with relatively simple structures would be given, considering some typical perturbations. Compared with existing methods, a finite time controller based on a dynamic sliding mode will be proposed based on a standard sliding mode surface; the advantage of a fast convergence rate and strong robustness would be combined in this proposed method.

The structure of this paper is constructed as follows: 1 . Section 1 describes the background and innovation of this paper; 2 . Section 2 gives the math models used in this paper; 3 . Section 3 describes the issue needs to be solved in this paper; 4 . Section 4 presents a finite-time controller based on attitude quaternion and proves some properties of this controller; 5 . Section 5 presents a finite-time controller based on the Euler axis and system performance is improved comparing with that in Section 4; 6. Section 6 demonstrates the controller performance by simulation results; 7 . Section 7 concludes the paper.

\section{Dynamics and Models}

The dynamic model of rigid satellite could be modeled as follows [4-7]:

$$
J \dot{\omega}+\omega^{\times} J \omega=u+d
$$


where $\omega$ is angular velocity which is a $3 \times 1$ vector, $J$ is inertia matrix of satellite which is a $3 \times 3$ positive definite symmetric matrix, $d$ is $3 \times 1$ unknown disturbance torque with norm upper bound $\|\boldsymbol{d}\|<\bar{d}$. Product matrix $\boldsymbol{r}^{\times}$of vector $\boldsymbol{r}$ is defined as

$$
\boldsymbol{r}^{\times}=\left[\begin{array}{ccc}
0 & -r_{3} & r_{2} \\
r_{3} & 0 & -r_{1} \\
-r_{2} & r_{1} & 0
\end{array}\right]
$$

generally inertia matrix $J$ could not be accurate known and it is assumed that

$$
J=\hat{J}+\widetilde{J}
$$

where $\hat{J}$ is the inertia matrix estimation and $\widetilde{J}$ is the error matrix. In this paper, the error matrix $\widetilde{J}$ could be treated as a disturbance in control system and one of the main goals is to design adaptive law to suppress this disturbance.

The kinetic model based on Euler axis / Angle could be written as follows [4-7]

$$
\left\{\begin{array}{c}
\dot{\boldsymbol{e}}=\frac{1}{2} \boldsymbol{e}^{\times}\left(\boldsymbol{I}_{3}-\cot \frac{\varphi}{2} \boldsymbol{e}^{\times}\right) \boldsymbol{\omega} \\
\dot{\varphi}=\frac{1}{2} \boldsymbol{e}^{T} \boldsymbol{\omega}
\end{array}\right.
$$

where $e$ is Euler axis and $\varphi$ is rotate angle. Based on (4) it could be found that kinetic model (4) has singularity issue when $\varphi \rightarrow 0$ i.e., the Euler axis $e$ is not continuous near the system equilibrium point.

The kinetic model based on attitude quaternion could be written as follows [4-7]

$$
\left\{\begin{array}{c}
\dot{q}_{0}=-\frac{1}{2} \boldsymbol{q}_{v}^{T} \boldsymbol{\omega} \\
\dot{\boldsymbol{q}}_{v}=\frac{1}{2}\left(q_{0} \boldsymbol{I}_{3}+\boldsymbol{q}_{v}^{\times}\right) \boldsymbol{\omega}=\frac{1}{2} \boldsymbol{F} \boldsymbol{\omega}
\end{array}\right.
$$

Considering that $\boldsymbol{q}$ and $-\boldsymbol{q}$ describes the same attitude, the scalar part of attitude quaternion is assumed to be non-negative in this paper i.e., $q_{0} \geq 0$.

\section{Problem Formulation}

In satellite attitude control issue, standard sliding mode could written as follows

$$
s=\omega+k q_{v^{\prime}}(k>0)
$$

when system converges along the sliding mode (11) it could be found that

$$
\begin{gathered}
\boldsymbol{\omega}=-k \boldsymbol{q}_{v} \\
\dot{\boldsymbol{q}}_{v}=\frac{1}{2}\left(q_{0} \boldsymbol{I}_{3}+\boldsymbol{q}_{v}^{\times}\right) \boldsymbol{\omega}=-\frac{1}{2} k q_{0} \boldsymbol{q}_{v}
\end{gathered}
$$

when system maneuvers along (6), angular velocity vector is reversed to attitude quaternion vector and lot of work have been done based on this sliding mode. The model uncertainty and unknown disturbance issue could be effectively solved using sliding mode (6) and it could be concluded that the reverse property could improve system robustness. However, based on equation (6) it could be easily found that the convergence rate of $\boldsymbol{q}_{v}$ is exponential which means system would reach the equilibrium point with infinite time and the convergence rate needs to be improved.

In order to improve system convergence rate, finite-time controller is an effective method. Generally in order to achieve the finite-time stability, fraction order feedback is used as follows to construct the sliding mode.

$$
\dot{\boldsymbol{x}}=-k \operatorname{sign}(\boldsymbol{x})|\boldsymbol{x}|^{r}, 0<r<1
$$


where $\operatorname{sign}(x)$ is the sign function of vector $x$.

Sliding mode (8) would bring another issue i.e., the singularity issue. Since the control torque is always related to $\ddot{x}$ i.e., the 2 nd derivative of $x$, the singularity term $x^{r-1}$ would be brought into the controller. In order to deal with the singularity issue some typical finitetime controllers are designed $[4,11,13]$. However, system robustness issue is not taken into consideration and the reverse property does not hold in these works. System robustness needs to be improved to suppress the perturbations such as inertia matrix uncertainty and unknown disturbance. In summary, the robustness issue and singularity issue should be both taken into consideration to design the robust finite-time controller.

Based on the discussion above, the goal of this paper could be as: design finite time controller for satellite stabilization issue and following properties should be satisfied:

1. Comparing with standard sliding mode, system convergence rate near the equilibrium point should be largely improved;

2. Finite-time stability should be satisfied i.e., there exist positive scalar $\varepsilon$ and $T$ to satisfy $\left\|\boldsymbol{q}_{v}\right\| \leq \varepsilon$ for $\forall t \geq T$;

3. The singularity issue should be solved i.e., $\boldsymbol{q}_{v}, \dot{\boldsymbol{q}}_{v^{\prime}}, \boldsymbol{\omega}, \dot{\boldsymbol{\omega}}$ are all bounded during the whole control process;

4. The controller should be robust to inertia matrix uncertainty and unknown disturbance torque.

\section{Finite-Time Controller Based on Attitude Quaternion}

In paper [5], the author pointed out that the fixed sliding mode caused the low convergence rate and a dynamic sliding mode is constructed in this paper. The maneuver stage with constant angular velocity and converge stage with a constant angular acceleration is designed based on the update law of sliding mode parameter $k$, and the system convergence rate is largely improved when compared with the standard sliding mode. Inspired by the method in [5], the finite-time sliding mode proposed in this paper could written as follows:

$$
\begin{gathered}
\boldsymbol{s}=\boldsymbol{\omega}+k \boldsymbol{q}_{v} \\
\dot{k}=\left\{\begin{array}{cc}
0 & \|\boldsymbol{s}\|>\varepsilon_{1} \\
\frac{k}{2}(1-\alpha) \beta q_{0}\left\|\boldsymbol{q}_{v}\right\|^{\alpha-1} & \|\boldsymbol{s}\| \leq \varepsilon_{1}
\end{array}\right. \\
1 / 2<\alpha<1, \beta=k\left(t_{0}\right) /\left\|\boldsymbol{q}_{v}\left(t_{0}\right)\right\|^{\alpha-1}
\end{gathered}
$$

where the initial value of $k$ and satisfies $k\left(t_{0}\right)>0, \varepsilon_{1}$ is a small positive scalar, $\alpha, \beta$ are all positive scalars.

Sliding mode (9) has the same structure as standard sliding mode hence the reversed property could be maintained. Moreover, the same structure could make it possible to design a robust finite-time controller based on standard sliding mode methods. Based on (9) it could be found that the maneuver process is constructed as two stages: in the first stage i.e., $\|\boldsymbol{s}\|>\varepsilon_{1}$, system performance is totally same as that of standard sliding mode, and sliding mode parameter $k$ is fixed; in the second stage i.e., $\|\boldsymbol{s}\| \leq \varepsilon_{1}$, it could be treated that system has reached the sliding mode and angular velocity vector has been reversed to attitude quaternion vector. In this stage, sliding mode parameter $k$ begins to update. Moreover, based on the update law of $k$ it could be found that $k$ is monotonically increasing to effect the exponential convergence rate. The key work of this paper is the update law of sliding mode parameter $k$ and when system convergences along (9) i.e., $s=0$, system (5) would converges to its equilibrium point within finite time, and during this process $\omega$ and $\dot{\omega}$ are all norm upper bounded. 
First the finite-time stability on sliding mode (9) is discussed. When system reaches sliding mode (9), define as follows and calculate its derivative it could be got that

$$
\begin{gathered}
V_{q}=\boldsymbol{q}_{v}^{T} \boldsymbol{q}_{v}=\left\|\boldsymbol{q}_{v}\right\|^{2} \\
\dot{V}_{q}=2 \boldsymbol{q}_{v}^{T} \dot{\boldsymbol{q}}_{v}=-k q_{0} \boldsymbol{q}_{v}^{T} \boldsymbol{q}_{v}=-k q_{0}\left\|\boldsymbol{q}_{v}\right\|^{2}
\end{gathered}
$$

In order to achieve the goal of finite-time stability, the derivative of Lyapunov function should satisfy following inequality

$$
\dot{V}_{q} \leq-\gamma q_{0}\left\|\boldsymbol{q}_{v}\right\|^{\alpha+1} \text {, with } \alpha \in(0,1), \gamma>0
$$

Comparing with (12) and (13) it could be got that if there exist positive scalar $\gamma$ to satisfy following inequality, the finite-time stability could be ensured.

$$
k=\gamma\left\|\boldsymbol{q}_{v}\right\|^{\alpha-1}
$$

In order to satisfy finite-time condition (14), fixed parameter $k$ is not feasible since the right part of (14) tends to infinite, and a very large $k$ would cause the control torque an angular velocity exceed system upper bound drastically. Hence it is necessary to design a time-variable parameter $k$ and its update law to satisfy (14) and that is how the dynamic sliding mode (9) is got. In fact, select parameters as follows, it could be got that

$$
\gamma=k\left(t_{0}\right) /\left\|\boldsymbol{q}_{v}\left(t_{0}\right)\right\|^{\alpha-1}, \beta=\gamma
$$

Noticing that the structure of sliding mode parameter update law in (9), it could be got that

$$
\begin{gathered}
k\left(t_{0}\right)=\gamma\left\|\boldsymbol{q}_{v}\left(t_{0}\right)\right\|^{\alpha-1} \\
\dot{k}=\frac{1}{2} k(1-\alpha) \beta q_{0}\left\|\boldsymbol{q}_{v}\right\|^{\alpha-1}=\frac{1}{2} k\left(1-\alpha_{0}\right) \gamma q_{0}\left\|\boldsymbol{q}_{v}\right\|^{\alpha-1}=\frac{d \gamma\left\|\boldsymbol{q}_{v}\right\|^{\alpha-1}}{d t}
\end{gathered}
$$

Based on (15) and (16) it could be found that finite-time condition (14) is satisfied, and (12) could be transformed to

$$
\dot{V}_{q}=2 \boldsymbol{q}_{v}^{T} \dot{\boldsymbol{q}}_{v}=-k q_{0} \boldsymbol{q}_{v}^{T} \boldsymbol{q}_{v}=-k q_{0}\left\|\boldsymbol{q}_{v}\right\|^{2} \leq-\beta q_{0}\left\|\boldsymbol{q}_{v}\right\|^{\alpha+1}=-\beta q_{0} V_{q}^{\alpha+1 / 2}
$$

System converge time satisfies

$$
t_{f} \leq \frac{2 V^{\frac{1-\alpha}{2}}\left(t_{0}\right)}{\beta q_{0}\left(t_{0}\right)(1-\alpha)}
$$

The next step is to prove on sliding mode (9), $\omega, \dot{\omega}$ are all norm upper bounded. It is obviously that angular velocity $\omega$ satisfies following property and is norm upper bounded.

$$
\|\boldsymbol{\omega}\|=\left\|-k \boldsymbol{q}_{v}\right\|=\left\|\boldsymbol{q}_{v}\right\|^{\alpha}
$$

Calculate the derivative of angular velocity $\omega$ it could be got that

$$
\begin{aligned}
\dot{\boldsymbol{\omega}} & =-k \dot{\boldsymbol{q}}_{v}-\dot{k} \boldsymbol{q}_{v} \\
& =-k\left(q_{0} \boldsymbol{I}_{3}+\boldsymbol{q}_{v}^{\times}\right)\left(-k \boldsymbol{q}_{v}\right)-\frac{k}{2}(1-\alpha) \beta q_{0}\left\|\boldsymbol{q}_{v}\right\|^{\alpha-1} \boldsymbol{q}_{v} \\
& =q_{0} k^{2} \boldsymbol{q}_{v}-\frac{k}{2}(1-\alpha) \beta q_{0}\left\|\boldsymbol{q}_{v}\right\|^{\alpha-1} \boldsymbol{q}_{v} \\
& =q_{0} \beta^{2}\left\|\boldsymbol{q}_{v}\right\|^{2 \alpha-1} \boldsymbol{e}-\frac{1}{2}(1-\alpha) \beta^{2} q_{0}\left\|\boldsymbol{q}_{v}\right\|^{2 \alpha-1} \boldsymbol{e}
\end{aligned}
$$


Consider that $1 / 2<\alpha<1$, hence $\omega, \dot{\omega}$ are all norm upper bounded during the whole maneuver process, and the demand control torque is also norm upper bounded i.e., the singularity issue is solved.

Based on the discussion above it could be found that the system state on the sliding mode (9) is norm upper bounded, however according to the update law of $k$ it could be found that sliding mode parameter $k$ tends to infinity as the system convergence. Although the system state and control actuator would not be influenced by this divergence, the computation system would break down under sliding mode (9). Hence for engineering practice, finite-time sliding mode (9) could be re-written as follows

$$
\begin{gathered}
\boldsymbol{s}=\boldsymbol{\omega}+k \boldsymbol{q}_{v} \\
0 \\
\dot{k}=\left\{\begin{array}{cc}
\|\boldsymbol{s}\|>\varepsilon_{1} \\
\frac{k}{2}(1-\alpha) \beta q_{0}\left\|\boldsymbol{q}_{v}\right\|^{\alpha-1} & \|\boldsymbol{s}\| \leq \varepsilon_{1},\left\|\boldsymbol{q}_{v}\right\|>\varepsilon_{2} \\
0 & \|\boldsymbol{s}\| \leq \varepsilon_{1},\left\|\boldsymbol{q}_{v}\right\| \leq \varepsilon_{2}
\end{array}\right.
\end{gathered}
$$

where $\varepsilon_{1}$ and $\varepsilon_{2}$ are all small positive scalars. It could be found that the basic structure of (21) is the same as (9), hence the system's finite-time stability and bounded state property could be maintained, and the only difference is that when the system approaches the equilibrium point, the sliding mode parameter stops updating to avoid the parameter singularity issue.

The closed control loop scheme block diagram is shown as follows.

Shown as Figure 1, the control system is constructed as a sliding mode surface, controller and update law. The latest one is the main contribution of this paper and it will be described in the text.

System Input

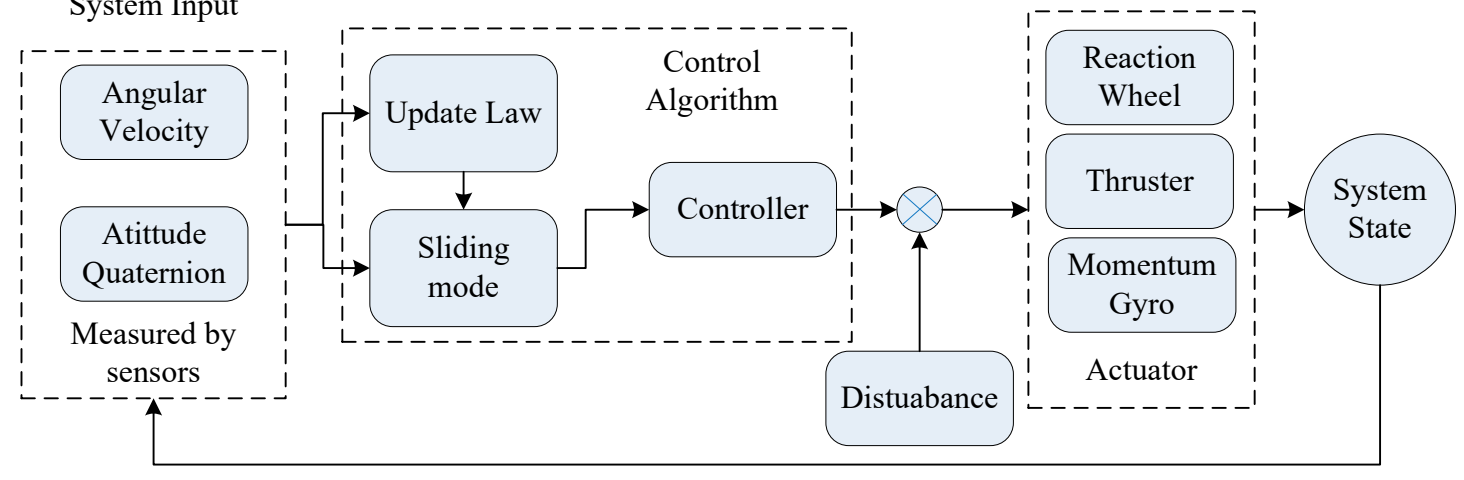

Figure 1. Control block diagram.

The next step is to propose the attitude controller after giving the sliding mode (21). The robust finite-time controller proposed in this paper based on (21) could be written as follows

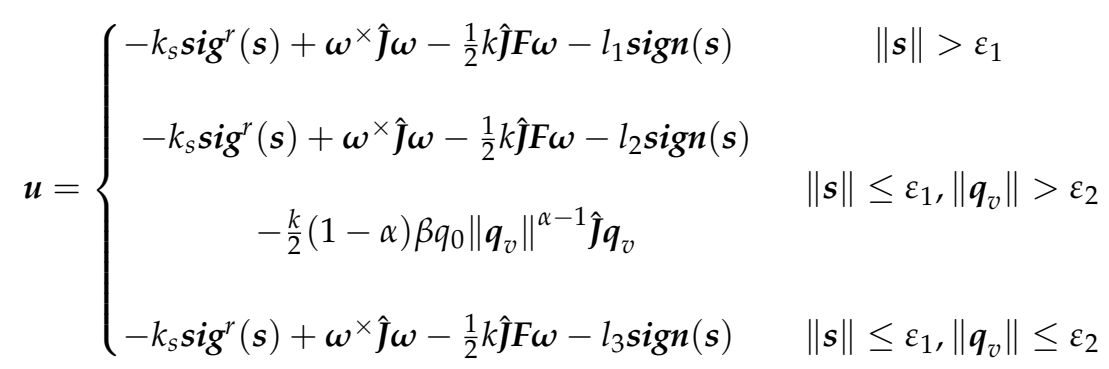


where $k_{s}$ is a positive scalar, $r$ is a positive scalar which satisfies $0<r<1$, vector function $\operatorname{sig}^{r}(\boldsymbol{x})$ and $l_{i}$ are defined as follows

$$
\begin{gathered}
\operatorname{sig}^{r}(\boldsymbol{x})=\boldsymbol{x} /\|\boldsymbol{x}\|^{r} \\
\left\{\begin{array}{c}
l_{1}=\bar{d}+\lambda\|\boldsymbol{\omega}\|^{2}+\frac{k}{2} \lambda\|\boldsymbol{\omega}\| \\
l_{2}=\bar{d}+\lambda\|\boldsymbol{\omega}\|^{2}+\frac{k}{2} \lambda\|\boldsymbol{\omega}\|+\frac{k}{2} \lambda(1-\alpha) \beta q_{0}\left\|\boldsymbol{q}_{v}\right\|^{\alpha} \\
l_{3}=\bar{d}+\lambda\|\boldsymbol{\omega}\|^{2}+\frac{k}{2} \lambda\|\boldsymbol{\omega}\|
\end{array}\right.
\end{gathered}
$$

where $\lambda$ is a positive scalar which satisfies $\lambda \geq \lambda_{M}(\widetilde{J})$ with $\lambda_{M}(\widetilde{J})$ is the maximum eigenvalue value of error inertia matrix $\widetilde{J}$.

Controller (22) has the three following properties: (1) there is no negative power term of system state in controller (22) hence the control torque are norm upper-bounded during the whole control process; (2) as the system state converges, most sign function terms in $l_{i}$ tends to zero hence at the steady stage the actual sign function term is $\bar{d}$ to suppress the disturbance torque; and (3) the system inertia matrix uncertainty is treated as king of disturbance related to system state with norm upper bound and suppressed by sign function terms, hence controller (23) is robust to model uncertainty.

The next step is to prove that governed by controller (22), system could reach sliding mode (21) within finite-time. Select Lyapunov function as follows

$$
V_{s}=\frac{1}{2} s^{T} J s
$$

The $V$ function satisfies following property

$$
V \geq \frac{1}{2} \lambda_{m}(J)\|s\|^{2}
$$

where $\lambda_{m}(J)$ is the minimum eigenvalue value of matrix $J$.

When $\|\boldsymbol{s}\|>\varepsilon_{1}$ and $\|\boldsymbol{s}\| \leq \varepsilon_{1},\left\|\boldsymbol{q}_{v}\right\| \leq \varepsilon_{2}$, calculate the derivative of $V$ function and noticing (25) it could be got that

$$
\begin{aligned}
\dot{V}_{s} & =s^{T} J \dot{s}=s^{T} J \dot{\omega}+k s^{T} J \dot{q}_{v} \\
& =s^{T} u-s^{T} \omega^{\times} J \omega+s^{T} d+\frac{k}{2} s^{T} J F \omega \\
& =k_{s} s^{T} \operatorname{sig}^{r}(s)-s^{T} \omega^{\times} \widetilde{J} \omega+s^{T} d+\frac{k}{2} s^{T} \widetilde{J} \boldsymbol{\omega} \\
& -\left(\bar{d}+\lambda\|\omega\|^{2}+\frac{k}{2} \lambda\|\omega\|\right) s^{T} \operatorname{sign}(s) \\
& \leq-k_{s}\|s\|^{r+1}+\bar{d}\|s\|+\lambda_{M}(\widetilde{J})\|s\|\|\omega\|^{2}+\frac{k}{2} \lambda_{M}(\widetilde{J})\|s\|\|\omega\| \\
& -\left(\bar{d}+\lambda\|\omega\|^{2}+\frac{k}{2} \lambda\|\omega\|\right)\|s\|-\bar{d}\|s\| \\
& \leq-k_{s}\|s\|^{r+1} \leq-\mu V^{\frac{r+1}{2}}
\end{aligned}
$$

When $\|s\| \leq \varepsilon_{1},\left\|\boldsymbol{q}_{v}\right\|>\varepsilon_{2}$ calculate the derivative of $V$ function and noticing (25) it could be got that 


$$
\begin{aligned}
& \dot{V}_{s}=s^{T} J \dot{s}=s^{T} J \dot{\omega}+k s^{T} J \dot{q}_{v}+\dot{k s}^{T} J q_{v} \\
& =s^{T} u-s^{T} \omega^{\times} J \omega+s^{T} d+\frac{k}{2} s^{T} J F \omega+k^{T} J q_{v} \\
& =k_{s} s^{T} s i g^{r}(s)-s^{T} \omega^{\times} \widetilde{J} \omega+s^{T} d+\frac{k}{2} s^{T} \widetilde{J} F \omega+\dot{k}^{T} \widetilde{J} q_{v} \\
& -\left(\bar{d}+\lambda\|\boldsymbol{\omega}\|^{2}+\frac{k}{2} \lambda\|\boldsymbol{\omega}\|+\dot{k} \lambda\left\|\boldsymbol{q}_{v}\right\|\right) \boldsymbol{s}^{T} \operatorname{sign}(\boldsymbol{s}) \\
& \leq-k_{s}\|\boldsymbol{s}\|^{r+1}+\bar{d}\|\boldsymbol{s}\|+\lambda_{M}(\widetilde{J})\|\boldsymbol{s}\|\|\boldsymbol{\omega}\|^{2}+\frac{k}{2} \lambda_{M}(\widetilde{J})\|\boldsymbol{s}\|\|\boldsymbol{\omega}\|+\dot{k} \lambda_{M}(\widetilde{\boldsymbol{J}})\|\boldsymbol{s}\|\left\|\boldsymbol{q}_{v}\right\| \\
& -\left(\bar{d}+\lambda\|\boldsymbol{\omega}\|^{2}+\frac{k}{2} \lambda\|\boldsymbol{\omega}\|+\dot{k} \lambda\left\|\boldsymbol{q}_{v}\right\|\right)\|\boldsymbol{s}\|-\bar{d}\|\boldsymbol{s}\| \\
& \leq-k_{s}\|s\|^{r+1} \leq-\mu V^{\frac{r+1}{2}}
\end{aligned}
$$

In (26) and (27), parameter $\mu$ is defined as follows

$$
\mu=k_{S}\left(2 / \lambda_{m}(J)\right)^{\frac{r+1}{2}}
$$

Based on (26) and (27) it could be found that system (1), (5) governed by controller (22) could reach finite-time sliding mode (21) within finite-time, and along this sliding mode system would converge to $\left\|\boldsymbol{q}_{v}\right\| \leq \varepsilon_{2}$ within finite-time.

In this section, a finite-time sliding mode based on standard sliding mode is proposed. The basic structure, physical meaning and inherent robustness of the standard sliding mode could be maintained, and the fixed parameter is modified to time-varying with the typical update law. The strict finite-time time stability could be achieved by updating the sliding mode parameter to infinite without causing the singularity issue of the system state, however the computation system would break down by this method. Hence the finite-time stability is loosed and when system state approaches to its equilibrium point close enough, the parameter stops updating.

\section{Finite-Time Controller Based on Euler Axis}

In the last section, in order to avoid the singularity issue of the sliding mode parameter, the system lost its strict finite-time stability. Hence, this section will discuss another finitetime sliding mode based on the standard sliding mode to achieve strict finite-time stability without causing any singularity issues.

According to the description in Section 2, the Euler axis could also be used to describe attitude information, and is related to attitude quaternion tightly. However, this description has its own singularity issue i.e., when $\varphi \rightarrow 0, \dot{e} \rightarrow \infty$. The Euler axis is a unit vector and describes the direction of the attitude quaternion; a small change in attitude quaternion would cause a huge change in its direction when the system approaches its equilibrium point. This would explain why, when the Euler angle tends to zero, the kinetic model of the Euler axis is not continuous, and this property causes a huge challenge to design controllers based on the Euler axis/angle. However, noticing that when angular velocity vector is reversed to attitude quaternion vector i.e.,

$$
\omega=-k_{1} \boldsymbol{q}_{v}=-k_{2} e
$$

The kinetic model of Euler axis could be transformed to

$$
\dot{\boldsymbol{e}}=\frac{1}{2} \boldsymbol{e}^{\times}\left(I_{3}-\cot \frac{\varphi}{2} \boldsymbol{e}^{\times}\right) \omega=-\frac{1}{2} k_{2} \boldsymbol{e}^{\times}\left(I_{3}-\cot \frac{\varphi}{2} \boldsymbol{e}^{\times}\right) \boldsymbol{e}=0
$$

It could be found that the singularity issue does not exist in this condition. In essence, when angular velocity vector parallels to attitude quaternion vector, the direction of the 
Euler axis is constant. Based on this property, the sliding mode based on the Euler axis could be constructed as two stages: (1) the system angular velocity vector should be reversed to attitude quaternion vector; and (2) maintain the reverse property and adjust the norm of angular velocity to achieve strict finite-time stability. Hence the finite-time sliding mode proposed in this section could be written as follows

$$
\begin{gathered}
\boldsymbol{s}=\boldsymbol{\omega}+k \boldsymbol{e} \\
\dot{k}=\left\{\begin{array}{cc}
0 & \|\boldsymbol{s}\|>\varepsilon_{1} \\
-\frac{1}{2} q_{0} \alpha \beta k\left\|\boldsymbol{q}_{v}\right\|^{\alpha-1}-\gamma_{1} g-\gamma_{2} \operatorname{sign}(g)|g|^{\alpha_{0}} & \|s\| \leq \varepsilon_{1}
\end{array}\right.
\end{gathered}
$$

where $\varepsilon_{1}$ is a small positive scalar, $k\left(t_{0}\right)$ is the initial value of $k$ and is a positive scalar, $\beta$ is a positive scalar, $\alpha$ satisfies $1 / 2<\alpha<1, \alpha_{0}$ satisfies $0<\alpha<1, \gamma_{1}$ and $\gamma_{2}$ are all positive scalars, and parameter $g$ is defined as follows

$$
g=k-\beta\left\|\boldsymbol{q}_{v}\right\|^{\alpha}
$$

It could be found that the structure of sliding mode (31) is similar as (9) in the previous section; the first step is also to achieve the reverse of angular velocity and attitude quaternion, and during this process the sliding mode parameter is fixed. When the reverse property has been satisfied, the sliding mode parameter begins to update to achieve strict finite-time stability.

It is worth noting that when maneuvering along the sliding mode (31), the update law, $k \rightarrow \beta\left\|\boldsymbol{q}_{v}\right\|^{\alpha}$ could be achieved within finite-time. In fact, select the Lyapunov function as follows and calculate its derivative

$$
V_{g}=\frac{1}{2} g^{2}
$$

$$
\begin{aligned}
\dot{V}_{g} & =g \dot{g} \\
& =g\left(\dot{k}-\beta \frac{d\left\|\boldsymbol{q}_{v}\right\|}{d t}\right) \\
& =g\left(-\frac{1}{2} q_{0} \alpha \beta k\left\|\boldsymbol{q}_{v}\right\|^{\alpha-1}-\gamma_{1} g-\gamma_{2} \operatorname{sign}(g)|g|^{\alpha_{0}}+\frac{1}{2} q_{0} \alpha \beta k\left\|\boldsymbol{q}_{v}\right\|^{\alpha-1}\right) \\
& =-\gamma_{1} g^{2}-\gamma_{2} g^{\alpha_{0}+1} \\
& \leq-\gamma_{2} g^{\alpha_{0}+1}=-\gamma_{2}\left(2 V_{g}\right)^{\alpha_{0}+1 / 2}
\end{aligned}
$$

Considering the range of $\alpha_{0}$ it could be found that the error state system is finitetime stable i.e., $g \rightarrow 0$ could be achieved within finite-time, hence $k \rightarrow \beta\left\|\boldsymbol{q}_{v}\right\|^{\alpha}$ could be achieved within finite-time.

The next step is to discuss system stability on sliding mode (31). Define Lyapunov function as follows

$$
\boldsymbol{V}_{q}=\boldsymbol{q}_{v}^{T} \boldsymbol{q}_{v}=\left\|\boldsymbol{q}_{v}\right\|^{2}
$$

Calculate its derivative and noticing the relationship between $k$ and $\left\|\boldsymbol{q}_{v}\right\|$, it could be got that when near the equilibrium point

$$
\begin{aligned}
\dot{\boldsymbol{V}}_{q} & =2 \boldsymbol{q}_{v}^{T} \dot{\boldsymbol{q}}_{v}=\boldsymbol{q}_{v}^{T}\left(q_{0} \boldsymbol{I}_{3}+\boldsymbol{q}_{v}^{\times}\right)(-k \boldsymbol{e})=-k q_{0}\left\|\boldsymbol{q}_{v}\right\| \\
& =-q_{0} \beta\left\|\boldsymbol{q}_{v}\right\|^{\alpha+1} \leq q_{0} \beta V_{q}^{\alpha+1 / 2}
\end{aligned}
$$

Hence system has strict finite-time stability on sliding mode (31). Moreover, noticing that

$$
k=\beta\left\|\boldsymbol{q}_{v}\right\|^{\alpha}, \dot{k}=-q_{0} \alpha \beta^{2}\left\|\boldsymbol{q}_{v}\right\|^{2 \alpha-1}
$$


Noticing that $1 / 2<\alpha<1$, hence

$$
\begin{gathered}
k=\beta\left\|\boldsymbol{q}_{v}\right\|^{\alpha} \rightarrow 0 \\
\|\boldsymbol{\omega}\|=\|-k \boldsymbol{e}\|=\beta\left\|\boldsymbol{q}_{v}\right\|^{\alpha} \rightarrow 0 \\
\|\dot{\boldsymbol{\omega}}\|=\|-k \dot{\boldsymbol{e}}-\dot{k} \boldsymbol{e}\|=q_{0} \alpha \beta^{2}\left\|\boldsymbol{q}_{v}\right\|^{2 \alpha-1} \rightarrow 0
\end{gathered}
$$

This means that on sliding mode (31) system state and sliding mode parameter has no singularity issue during the whole convergence process. Compared to the finite-time sliding mode in the previous section, the sliding mode (31) maintains the strict finite-time stability and solves the parameter singularity issue. Since the norm of the Euler axis is bounded to 1 , a convergent parameter $k$ could achieve the finite-time goal without causing the singularity issue, and this is a main contribution of this paper. Moreover, the basic structure of standard sliding mode is maintained in this section, and the physical meaning and inherent robustness could be maintained in (31).

As discussed above, in order to avoid the inherent kinetic model singularity of Euler axis, an important assumption should be made: when approaching the equilibrium point, angular velocity vector has been reversed to attitude quaternion vector, i.e., for a small positive scalar $\varepsilon_{2}$, when $\left\|\boldsymbol{q}_{v}\right\| \leq \varepsilon_{2}$ is satisfied, $\boldsymbol{\omega}=-k \boldsymbol{e}$ has been satisfied. It is worth noticing that except for some small angle maneuver, this assumption could be achieved since the initial Euler angle is relatively large, hence this assumption is reasonable.

The finite-time controller based on sliding mode (31) could be written as follows

$$
\boldsymbol{u}= \begin{cases}-k_{s} \operatorname{sig}^{r}(s)+\omega^{\times} \hat{J} \omega-\frac{1}{2} k \hat{J} G \omega-l_{1} \operatorname{sign}(s) & \|s\|>\varepsilon_{1} \\ -k_{s} \operatorname{sig}^{r}(s)+\omega^{\times} \hat{J} \omega-\dot{k} \hat{\jmath} \boldsymbol{e}-l_{2} \operatorname{sign}(s) & \|s\| \leq \varepsilon_{1}\end{cases}
$$

where the definition of vector function $\operatorname{sig}^{r}(\cdot)$ and sign function $\operatorname{sign}(\cdot)$ are totally same as previous section, $r$ is a positive scalar which satisfies $0<r<1, k_{s}$ is a positive scalar, the definition of $k$ is given in (33), matrix $G$ and scalars $l_{i}$ are defined as follows

$$
\begin{gathered}
G=e^{\times}\left(\boldsymbol{I}_{3}-\cot \frac{\varphi}{2} e^{\times}\right) \\
\left\{\begin{array}{c}
l_{1}=\bar{d}+\lambda\|\boldsymbol{\omega}\|^{2}+\frac{k}{2} \lambda\left(1+\cot \frac{\varphi}{2}\right)\|\boldsymbol{\omega}\| \\
l_{2}=\bar{d}+\lambda\|\boldsymbol{\omega}\|^{2}+\|\dot{k}\|
\end{array}\right.
\end{gathered}
$$

where $\lambda$ is a positive scalar which satisfies $\lambda \geq \lambda_{M}(\widetilde{J})$ with $\lambda_{M}(\widetilde{J})$ is the maximum eigenvalue value of error inertia matrix $\widetilde{J}$.

As discussed in previous section, controller (38) also has three properties: control torque norm upper bounded, most sign function terms tend to zero and robust to inertia matrix uncertainty.

Next step is to prove system (1) and (5) governed by controller (38) could reach the sliding mode (31) within finite-time. Select Lyapunov function as follows

$$
V_{s}=\frac{1}{2} s^{T} J s
$$

The $V$ function i.e., Lyapunov function satisfies

$$
V \geq \frac{1}{2} \lambda_{m}(J)\|s\|^{2}
$$


When $\|s\|>\varepsilon_{1}$, calculate the derivative of (43)

$$
\begin{aligned}
& \dot{V}_{S}=s^{T} J \dot{s}=s^{T} J \dot{\omega}+k s^{T} J \dot{e}+\dot{k s}^{T} J e \\
& =s^{T} u-s^{T} \omega^{\times} J \omega+s^{T} d+\frac{k}{2} s^{T} J G \omega \\
& =k_{S} s^{T} \operatorname{sig}^{r}(s)-s^{T} \omega^{\times} \widetilde{J} \omega+s^{T} d+\frac{k_{2}}{2} s^{T} \widetilde{J} G \omega \\
& -\left(\bar{d}+\lambda\|\omega\|^{2}+\frac{k}{2} \lambda\left(1+\cot \frac{\varphi}{2}\right)\|\omega\|\right) s^{T} \operatorname{sign}(s) \\
& \leq-k_{s}\|\boldsymbol{s}\|^{r+1}+\bar{d}\|\boldsymbol{s}\|+\lambda_{M}(\widetilde{\boldsymbol{J}})\|\boldsymbol{s}\|\|\boldsymbol{\omega}\|^{2}+\frac{k}{2} \lambda_{M}(\widetilde{J})\left(1+\cot \frac{\varphi}{2}\right)\|\boldsymbol{s}\|\|\boldsymbol{\omega}\| \\
& -\left(\bar{d}+\lambda\|\boldsymbol{\omega}\|^{2}+\frac{k_{2}}{2} \lambda\left(1+\cot \frac{\varphi}{2}\right)\|\boldsymbol{\omega}\|\right)\|\boldsymbol{s}\|-\bar{d}\|\boldsymbol{s}\| \\
& \leq-k\|s\|^{r+1} \leq-\mu V^{\frac{r+1}{2}}
\end{aligned}
$$

When $\|\boldsymbol{s}\| \leq \varepsilon_{1}$, calculate the derivative of (41) and noticing that angular velocity vector has been reversed to attitude quaternion vector i.e., $\dot{e}=0$

$$
\begin{aligned}
\dot{V}_{s} & =s^{T} \boldsymbol{J} \dot{\boldsymbol{s}}=\boldsymbol{s}^{T} \boldsymbol{J} \dot{\boldsymbol{\omega}}+k \boldsymbol{s}^{T} \boldsymbol{J} \dot{\boldsymbol{e}}+\dot{k}_{\boldsymbol{s}^{T}} \boldsymbol{J} \boldsymbol{e} \\
& =\boldsymbol{s}^{T} \boldsymbol{u}-\boldsymbol{s}^{T} \boldsymbol{\omega}^{\times} \boldsymbol{J} \boldsymbol{\omega}+\boldsymbol{s}^{T} \boldsymbol{d}+\dot{k s}^{T} \boldsymbol{J} \boldsymbol{e} \\
& =k_{s} \boldsymbol{s}^{T} \boldsymbol{s i g} \boldsymbol{g}^{r}(\boldsymbol{s})-\boldsymbol{s}^{T} \boldsymbol{\omega}^{\times} \widetilde{\boldsymbol{J}} \boldsymbol{\omega}+\boldsymbol{s}^{T} \boldsymbol{d}-q_{0} \alpha \beta^{2}\left\|\boldsymbol{q}_{v}\right\|^{2 \alpha-1} \boldsymbol{s}^{T} \widetilde{\boldsymbol{J}} \boldsymbol{e} \\
& -\left(\bar{d}+\lambda\|\boldsymbol{\omega}\|^{2}+\lambda q_{0} \alpha \beta^{2}\left\|\boldsymbol{q}_{v}\right\|^{2 \alpha-1}\right) \boldsymbol{s}^{T} \boldsymbol{s i g n}(\boldsymbol{s}) \\
& \leq-k_{s}\|\boldsymbol{s}\|^{r+1}+\bar{d}\|\boldsymbol{s}\|+\lambda_{M}(\widetilde{\boldsymbol{J}})\|\boldsymbol{s}\|\|\boldsymbol{\omega}\|^{2}+q_{0} \alpha \beta^{2} \lambda_{M}(\widetilde{\boldsymbol{J}})\left\|\boldsymbol{q}_{v}\right\|^{2 \alpha-1}\|\boldsymbol{s}\| \\
& -\left(\bar{d}+\lambda\|\boldsymbol{\omega}\|^{2}+\lambda q_{0} \alpha \beta^{2}\left\|\boldsymbol{q}_{v}\right\|^{2 \alpha-1}\right)\|\boldsymbol{s}\|-\bar{d}\|\boldsymbol{s}\| \\
& \leq-k\|\boldsymbol{s}\|^{r+1} \leq-\mu V^{\frac{r+1}{2}}
\end{aligned}
$$

In (42) and (43), parameter $\mu$ is defined as follows

$$
\mu=k\left(2 / \lambda_{m}(J)\right)^{\frac{r+1}{2}}
$$

Based on (43) and (44), it could be found that system (1) and (5) governed by controller (39) could reach finite-time sliding mode (31) within finite-time, and along this sliding mode the system would converge to the equilibrium point within finite-time; strict system finite-time stability has been proven.

In this section, the standard sliding mode is modified to have strict finite-time stability based on the Euler axis description. The property that norm of Euler axis is bounded is used to design the update law of sliding mode parameter, hence the system state and sliding mode parameter could be ensured norm upper bounded during the whole control process. Compared with the controller proposed in last section, the controller (39) has better convergence performance and robustness. However it is worth noticing that differs from last section, the control method in this section needs high attitude determination, since when approaching the system equilibrium point, a small error in attitude quaternion would cause a huge error in the Euler axis, thus the control accuracy would be influenced. In essence, finite-time attitude control issue is transformed to a high accuracy attitude determination issue. 


\section{Simulation}

In order to demonstrate the superiority of the adaptive finite-time controller (22) and (39) presented in this paper, the standard sliding mode controller (46) without the inertia matrix uncertainty constructed is compared as follows:

$$
\begin{gathered}
\boldsymbol{u}=-k \boldsymbol{s}+\boldsymbol{\omega}^{\times} \boldsymbol{J} \boldsymbol{\omega}-\frac{k_{1}}{2}\left(q_{0} \boldsymbol{I}_{3}+\boldsymbol{q}_{v}^{\times}\right) \boldsymbol{\omega}-\bar{d} \boldsymbol{s} \boldsymbol{g} \boldsymbol{n}(\boldsymbol{s}) \\
\boldsymbol{s}=\boldsymbol{\omega}+k_{1} \boldsymbol{q}_{v}
\end{gathered}
$$

Set the simulation parameters as follows

$$
\begin{aligned}
& J=\operatorname{diag}(30,25,20) \mathrm{kg} \cdot \mathrm{m}^{2}, k=0.1, k_{s}=10
\end{aligned}
$$

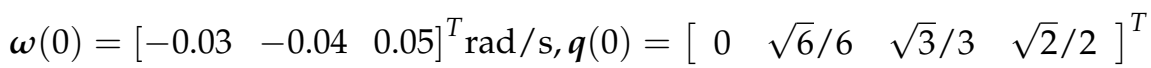

Assume the disturbance torque consists of Gauss white noise and sinusoidal signal written as follows

$$
d_{i}=5 \times 10^{-4} \operatorname{randn}(-1,1)+5 \times 10^{-4} \sin t+5 \times 10^{-4}\left\|\omega_{i}\right\| \operatorname{randn}(-1,1)
$$

Hence the norm upper bound of disturbance torque satisfies

$$
\bar{d}=10^{-3}
$$

The simulation results of standard sliding mode controller (48) are given as follows.

Based on Figures 2-4 it could be found that system converges to the equilibrium point more than $120 \mathrm{~s}$, and the steady accuracy at $150 \mathrm{~s}$ is about $1 \times 10^{-4} \mathrm{rad} / \mathrm{s}$ of angular velocity and $1 \times 10^{-3}$ of attitude quaternion. Based on the simulation parameters it could be found that the total rotate angle is $180 \mathrm{deg}$ and the maneuver time is longer than $120 \mathrm{~s}$, hence the average angular velocity is about $1.5 \mathrm{deg} / \mathrm{s}$. The low convergence rate is caused by the drop of angular velocity and this could be found in Figure 1. Moreover, based on Figure 3 it could be found that the initial control torque is about $1.5 \mathrm{Nm}$ and drops to zero drastically, hence it could concluded that the efficiency on control torque of standard sliding mode controller is relatively low.

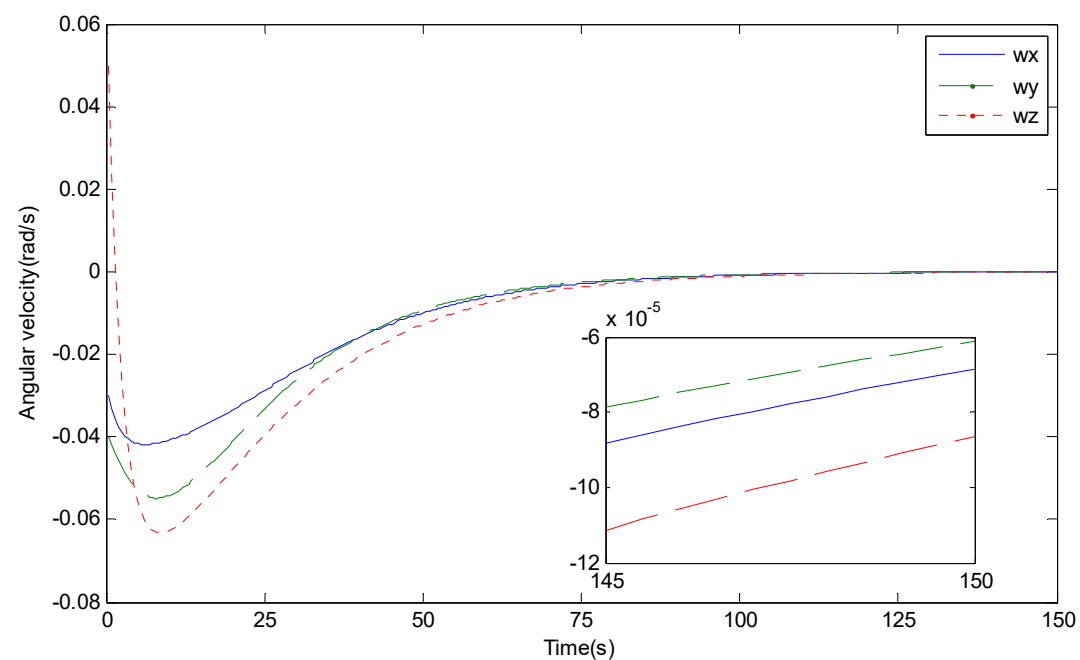

Figure 2. Curve of angular velocity. 


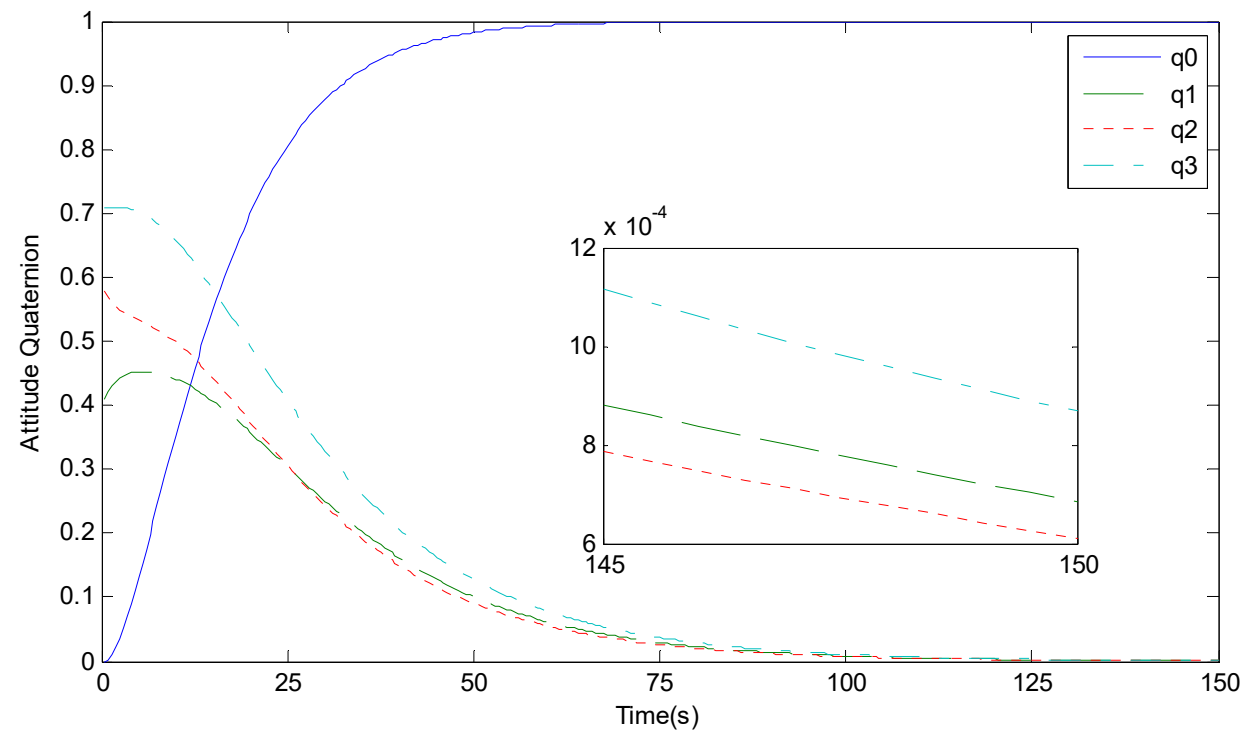

Figure 3. Curve of attitude quaternion.

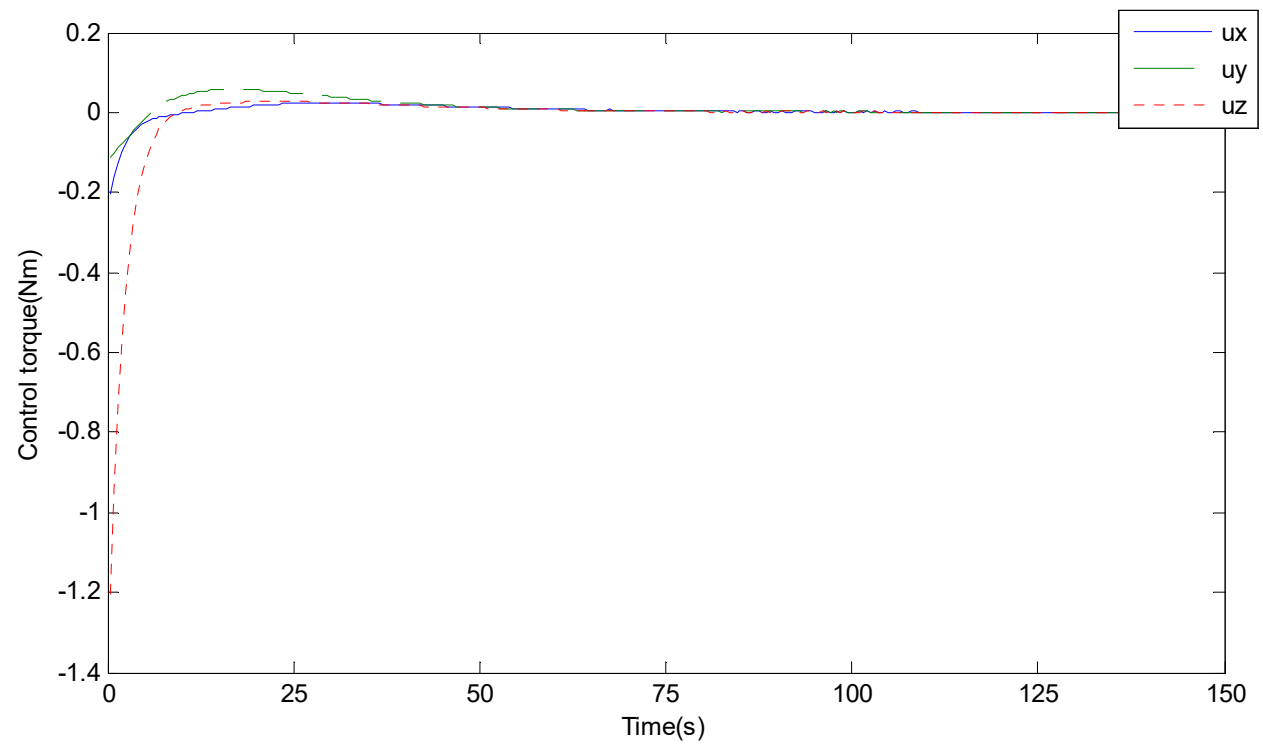

Figure 4. Curve of control torque.

\subsection{Simulation for Controller Based on Attitude Quaternion}

In this section, simulation results for finite-time controller (22) in Section 4 a given. Set system parameters as follows.

$$
\hat{J}=\operatorname{diag}(28,24,21) \mathrm{kg} \cdot \mathrm{m}^{2}, \lambda=3, \bar{d}=10^{-3}
$$

Generally, larger $r$ and $k\left(t_{0}\right)$ brings faster convergence rate, but the demanded control torque is also enlarged, and smaller $r$ makes sliding mode (9) degenerates to standard sliding mode. Also larger $k_{s}$ makes system could reach the finite-time sliding mode faster, moreover, smaller $\alpha$ and larger $\beta$ bring better convergence rate along the sliding mode. Considering that system performance under exponential convergence rate when away from the equilibrium point, the superiority of finite-time property mainly reflects on the 
performance near system equilibrium point. Above all, control parameters for controller (22) are selected as follows

$$
\begin{gathered}
\varepsilon_{1}=10^{-3}, \varepsilon_{2}=10^{-4}, r=1 / 3, k_{s}=2 \\
k\left(t_{0}\right)=0.1, \alpha=2 / 3, \beta=2
\end{gathered}
$$

Based on the discussion in previous section, system convergence time from initial condition to the field of $\left\|\boldsymbol{q}_{v}\right\| \leq 10^{-4}$ could be got as follows

$$
\begin{gathered}
T_{s} \leq 2 \frac{\left(V_{s}\left(t_{0}\right) / \varepsilon_{1}^{2}\right)^{1-r / 2}}{k_{s}\left(2 / \lambda_{m}(J)\right)^{r+1 / 2}(1-r)} \approx 14 \mathrm{~s}, T_{q} \leq 2 \frac{\left(1 / \varepsilon_{2}^{2}\right)^{1-\alpha / 2}}{\beta(1-\alpha)} \approx 44 \mathrm{~s} \\
T_{0} \leq T_{s}+T_{q}=58 \mathrm{~s}
\end{gathered}
$$

where $T_{s}$ is the time form initial state to the sliding mode, and $T_{q}$ is the convergence time along sliding mode (21).

The simulation results are shown as follows

Based on Figures 5 and 6 it could be found that system convergence time is about $30 \mathrm{~s}$, which is much larger than that of the standard sliding mode, also the finite-time stability calculated in (54) has been proved. The hsystem steady accuracy at $40 \mathrm{~s}$ is about $2 \times 10^{-6} \mathrm{rad} / \mathrm{s}$ of angular velocity and $4 \times 10^{-8}$ of attitude quaternion. System performance including convergence rate and steady accuracy is largely improved compared with that of standard sliding mode, and the superiority of the proposed controller in this paper is illustrated by simulation results. Based on Figures 5, 7 and 8 it could be found that the norm of angular velocity and control torque are upper bounded during the whole maneuver process, hence the singularity issue of finite-time control does not occur in the proposed controller. Based on Figure 7, it could be found that the updating sliding mode parameter $\mathrm{k}$ is the key to improve the system convergence rate. From the initial value to its terminal value, parameter $\mathrm{k}$ has enlarged more than 3000 times (from 0.1 to more than 30 ), and this property could offset the drawback brought by the exponential convergence rate. However, it is obvious that the curve of $k$ is very cliffy when the system state approaches the equilibrium point, and if the system continues to update parameter $k$, it would tend to infinity and cause the breakdown of the computing system. Moreover, noting the disturbance torque and model uncertainty in the simulation configuration, it could be concluded that controller (24) is robust to disturbance and model uncertainty. Above all, a finite-time controller (24) based on attitude quaternion proposed in this paper could achieve the goal of finite-time stability without causing the singularity issue, but the cost is that the finite-time stability is not strict (the system state could only reach the neighborhood of equilibrium point but not the actual equilibrium point within finite-time) to avoid the breakdown of computing system. 


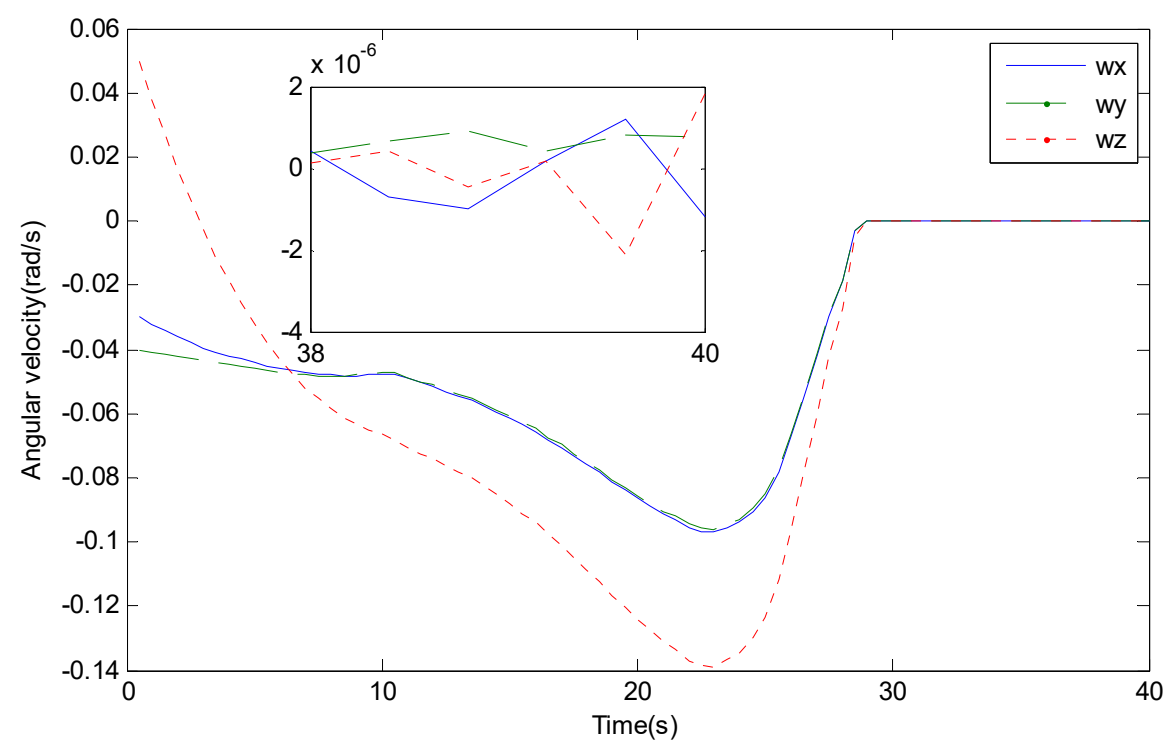

Figure 5. Curve of angular velocity.

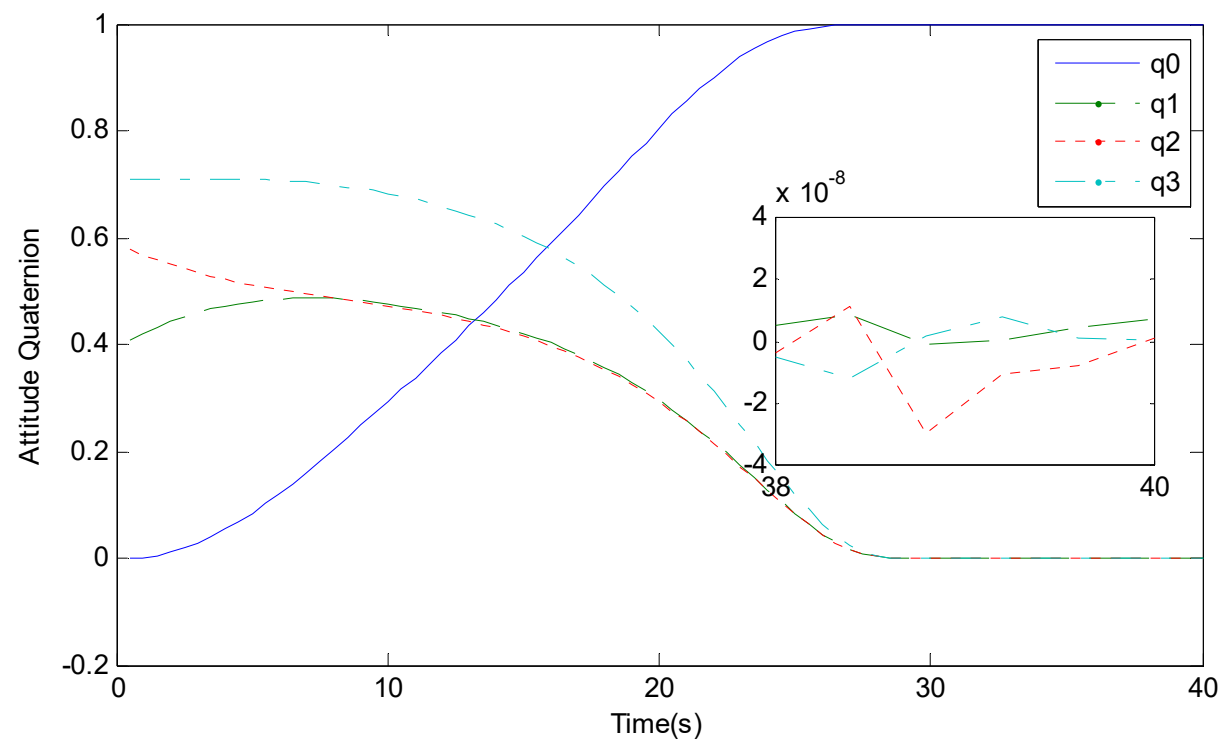

Figure 6. Curve of attitude quaternion. 


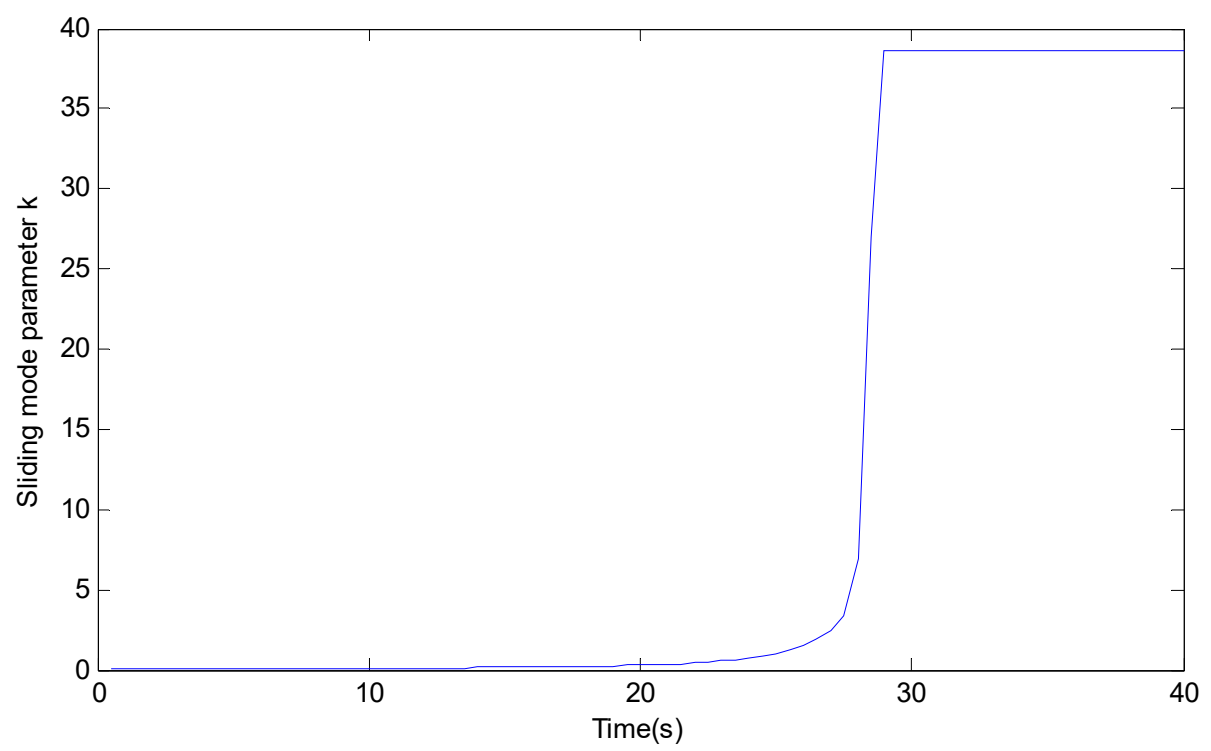

Figure 7. Curve of sliding mode parameter.

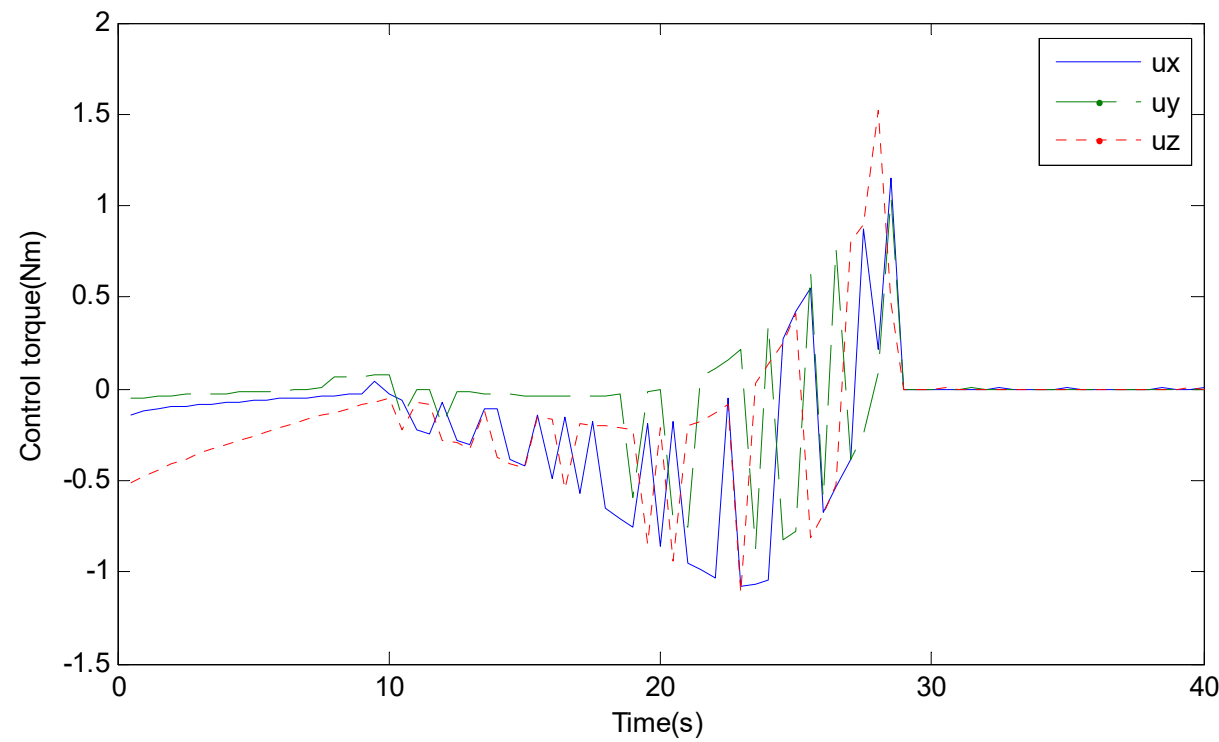

Figure 8. Curve of control torque.

Considering that the proposed sliding mode is modified based on standard sliding mode, hence some advantages such as the inherent robustness could be maintained. In order to demonstrate this property, set system configurations under controller (22) as follows

$$
\begin{gathered}
\boldsymbol{d}=1 \times 10^{-2} \times \operatorname{rand}(3 \times 1) \mathrm{Nm} \\
\bar{d}=0, \lambda=0, \boldsymbol{u}^{\prime}=\operatorname{diag}(0.9,0.8,0.7) \boldsymbol{u} \\
\boldsymbol{J}=\operatorname{diag}(30,25,20) \mathrm{kg} \cdot \mathrm{m}^{2}, \hat{\boldsymbol{J}}=\operatorname{diag}(22,18,15) \mathrm{kg} \cdot \mathrm{m}^{2}
\end{gathered}
$$

Based on (53) it could be found that the unknown disturbance torque is enlarged to the $10^{-2} \mathrm{Nm}$ level, and the term $\bar{d}$ to suppress disturbance in controller is set to be zero. Moreover, inertia matrix estimation has larger than $25 \%$ error comparing with actual inertia matrix, and the term $\lambda$ to suppress this perturbation is also set to be zero. The actual control output has constant bias from desired control torque. Above all, under this configuration system has three perturbation aspects: (1) larger disturbance and no offset 
term in controller; (2) larger model uncertainty and no offset term in controller; and (3) control output error in actuator.

Simulation results under condition (53) are show as follows.

Based on Figures 9 and 10 it could be found that the system could still converge to the equilibrium point under such perturbations. Comparing this group of simulation with Figures 5-8 it could be found that the main difference is the convergence time. Based on Figures 9-11 it could be found that system converge time is about $43 \mathrm{~s}$ and the convergence time of controller (22) is about $30 \mathrm{~s}$, and the steady accuracy could approximately be treated as the same level with controller (22). Moreover, Figures 11 and 12 demonstrate that finite-time sliding mode based on the standard sliding mode could resist some typical perturbations such as unknown disturbance, inertia matrix uncertainty and actuator error. Also, it is worth noting that although the demand control torque is discontinuous in Figure 12, it could be achieved by a reaction wheel, the function of which is to produce controlled torque by accelerating and decelerating its rotation speed, and this acceleration could be discontinuous. This proves that by designing the sliding mode properly, the finite-time stability and strong robustness could both be maintained.

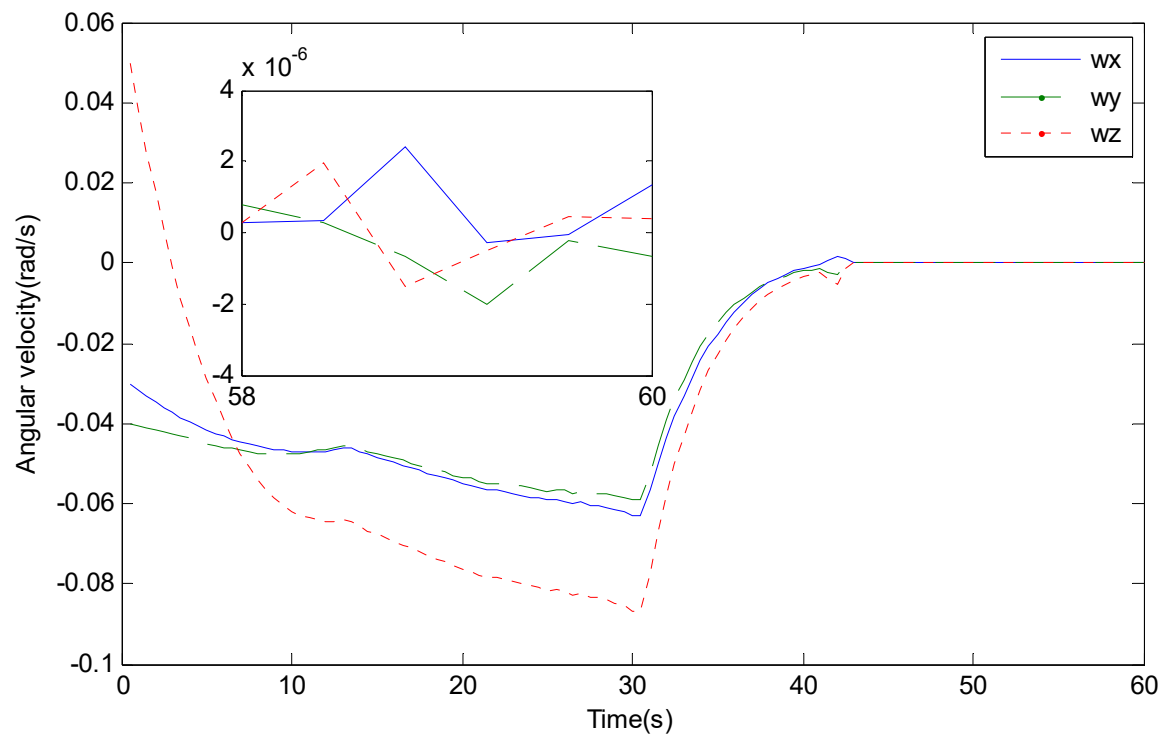

Figure 9. Curve of angular velocity.

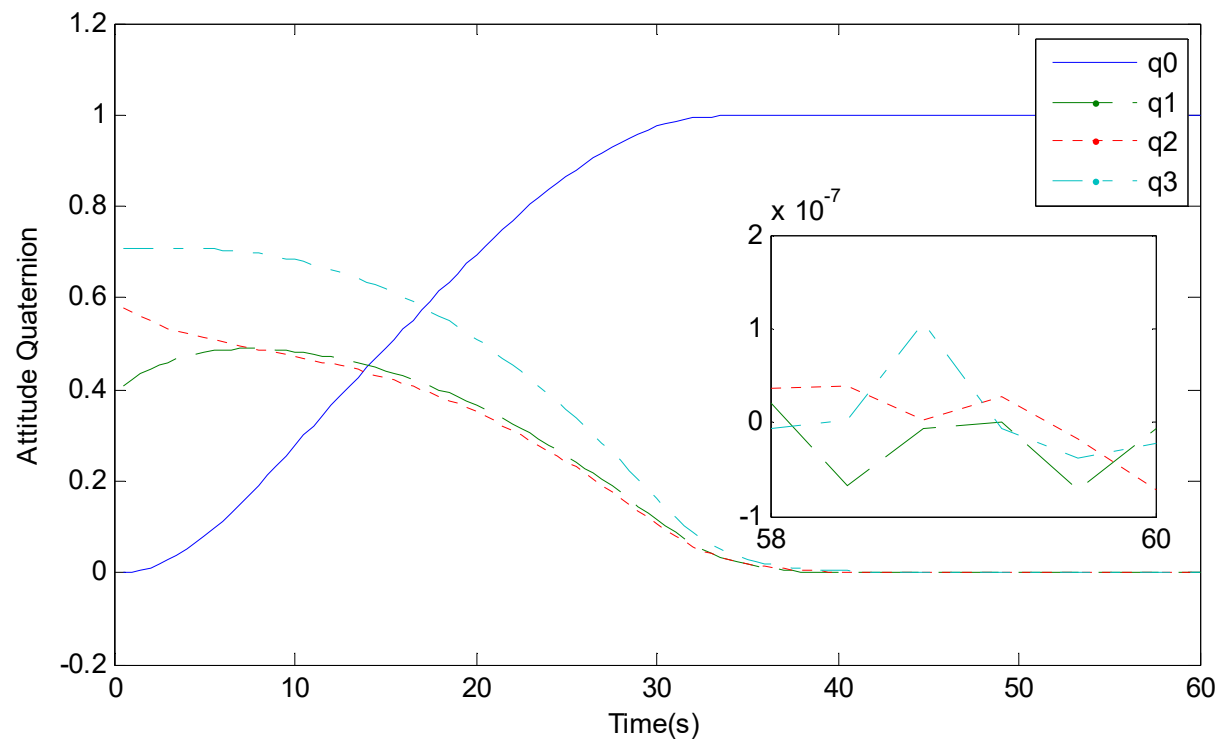

Figure 10. Curve of attitude quaternion. 


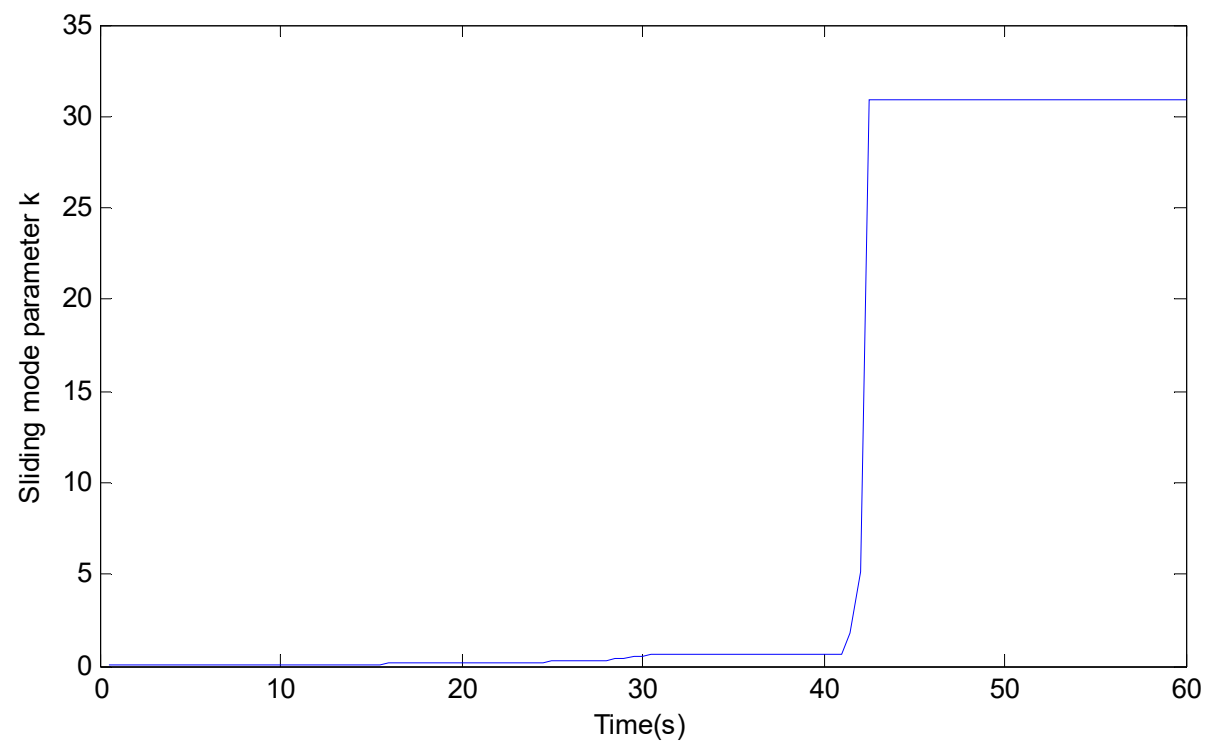

Figure 11. Curve of sliding mode parameter.

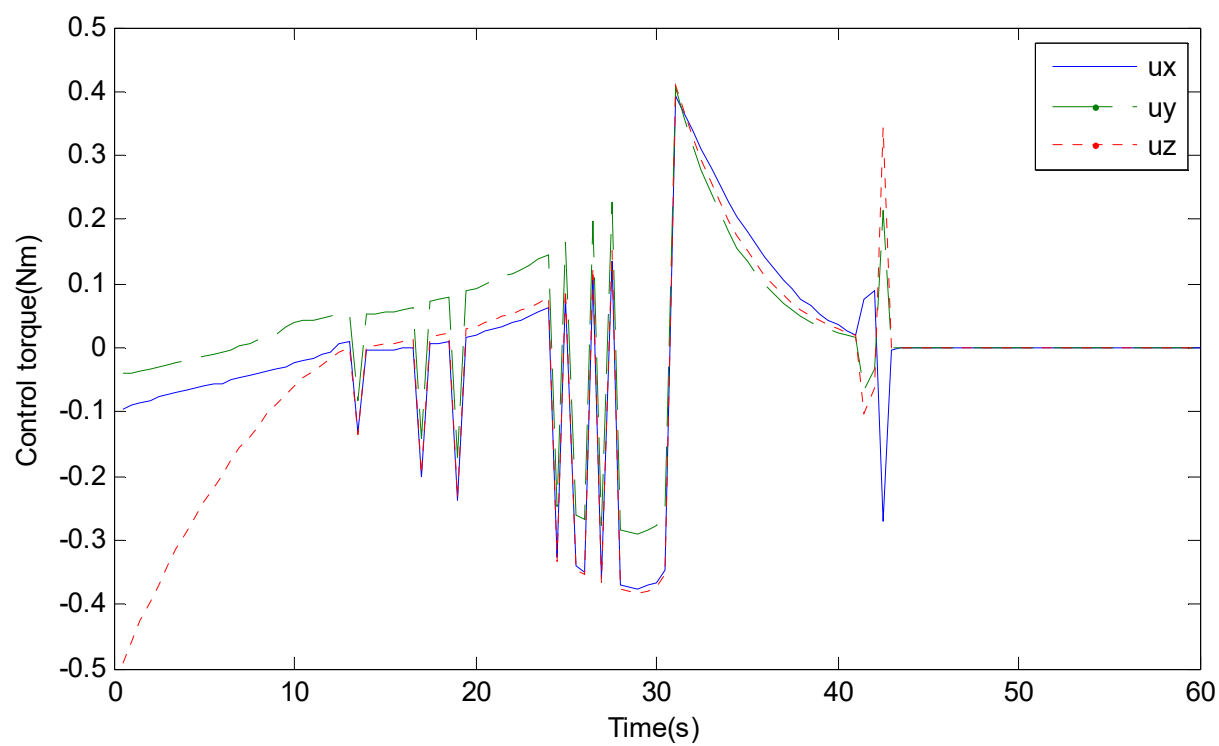

Figure 12. Curve of control torque.

In this section, simulation results for finite-time controller based on attitude quaternion are given. By the update law of sliding mode parameter, the finite-time stability could be ensured and the inherent robustness of the standard sliding mode could both be maintained. This is one of the main contributions of this paper and offers a new method to achieve finite-time stability. Also, it could be found that the parameter would tend to infinity and cause the breakdown of the computing system if the controller is not designed properly, hence the controller in Section 4 has some risk of the breakdown of the control system.

\subsection{Simulation for Controller Based on Euler Axis}

In this section, the simulation results of controller in Section 5 are given. Set system parameters as follows.

$$
\hat{J}=\operatorname{diag}(28,24,21) \mathrm{kg} \cdot \mathrm{m}^{2}, \lambda=3, \bar{d}=10^{-3}
$$

similar as discussed in Section 6.1, larger $r$ and $k\left(t_{0}\right)$ brings faster convergence rate, but the demanded control torque is also enlarged, and smaller $r$ makes sliding mode (9) degenerates 
to standard sliding mode. Also larger $k_{s}$ makes system could reach the finite-time sliding mode faster, moreover, smaller $\alpha$ and larger $\beta$ bring better convergence rate along the sliding mode. Larger $\gamma_{1}$ and $\gamma_{2}$ could ensure sliding mode parameter $k$ reach the desired trajectory. Considering the selection of control parameters in references [4-7], some control parameters could be selected similarly. Generally, larger sliding mode parameters could bring better convergence rates but the demanded control torque is also larger. Above all, control parameters for controller (39) are selected as follows

$$
\begin{gathered}
\varepsilon_{1}=10^{-4}, r=1 / 3, k_{s}=2, \gamma_{1}=\gamma_{2}=2 \\
k\left(t_{0}\right)=0.1, \alpha=2 / 3, \beta=1
\end{gathered}
$$

Assume that when $\left\|q_{v}\right\| \leq \varepsilon_{2}=10^{-4}$ is satisfied the system could be treated as converged to the equilibrium point, and the system convergence time could be calculated as follows:

$$
\begin{gathered}
T_{s} \leq 2 \frac{\left(V_{s}\left(t_{0}\right) / \varepsilon^{2}\right)^{1-r / 2}}{k_{s}\left(2 / \lambda_{m}(J)\right)^{r+1 / 2}(1-r)} \approx 12 \mathrm{~s}, T_{k} \leq 2 \frac{\left(\beta / k\left(t_{0}\right)\right)^{1-\alpha / 2}}{\beta(1-\alpha)} \approx 9 \mathrm{~s} \\
T_{q} \leq 2 \frac{\left(1 / \varepsilon_{2}^{2}\right)^{1-\alpha / 2}}{\beta(1-\alpha)} \approx 31 \mathrm{~s}, T_{0} \leq T_{s}+T_{k}+T_{q}=52 \mathrm{~s}
\end{gathered}
$$

where $T_{s}$ is the time form initial state to the sliding mode, $T_{k}$ is the time of $k$ chasing the desired trajectory, and $T_{q}$ is the convergence time along the proposed sliding mode.

The simulation results of controller (39) proposed in Section 5 is given as follows.

Based on Figures 13 and 14, it could be found that the system converges to the equilibrium point within $30 \mathrm{~s}$, and this proves the system finite-time stability calculated in Equation (56). Moreover, the system steady accuracy at $50 \mathrm{~s}$ is about $2 \times 10^{-5} \mathrm{rad} / \mathrm{s}$ of angular velocity and $6 \times 10^{-7}$ of attitude quaternion, which satisfy the converge condition claimed previously. Based on Figures 15 and 16, it could be found that control torque and sliding mode parameter are all norm upper bounded and the singularity issue does not occur in this condition. Comparing simulation results in this section with those in Section 6.1 it could be found that system convergence time is faster in this section, and the major improvement is the curve of sliding mode parameter $k$. In this section the sliding mode parameter tends to zero, hence it is not necessary to stop the update law of the sliding mode parameter. The robustness of the control system is strengthened by this property compared with the controller in Section 4. It is worth noting that when maneuvering along the proposed trajectory, the sliding mode parameter $k$ should be strictly monotonic decreasing. However, based on Figure 15, it could found that the parameter is not strictly monotonic decreasing and has some wave characters. This is caused by the discontinuous property of the Euler axis, since when approaching the equilibrium point, the Euler axis $e$ changes fast and is easily interfered with by random disturbance torque. Consider that under this situation, the changing rate of $\boldsymbol{e}$ tends to infinite and the controller could not offset this perturbation, hence the system state deviates from the desired trajectory until the control torque overwhelms the discontinuous perturbation torque. 


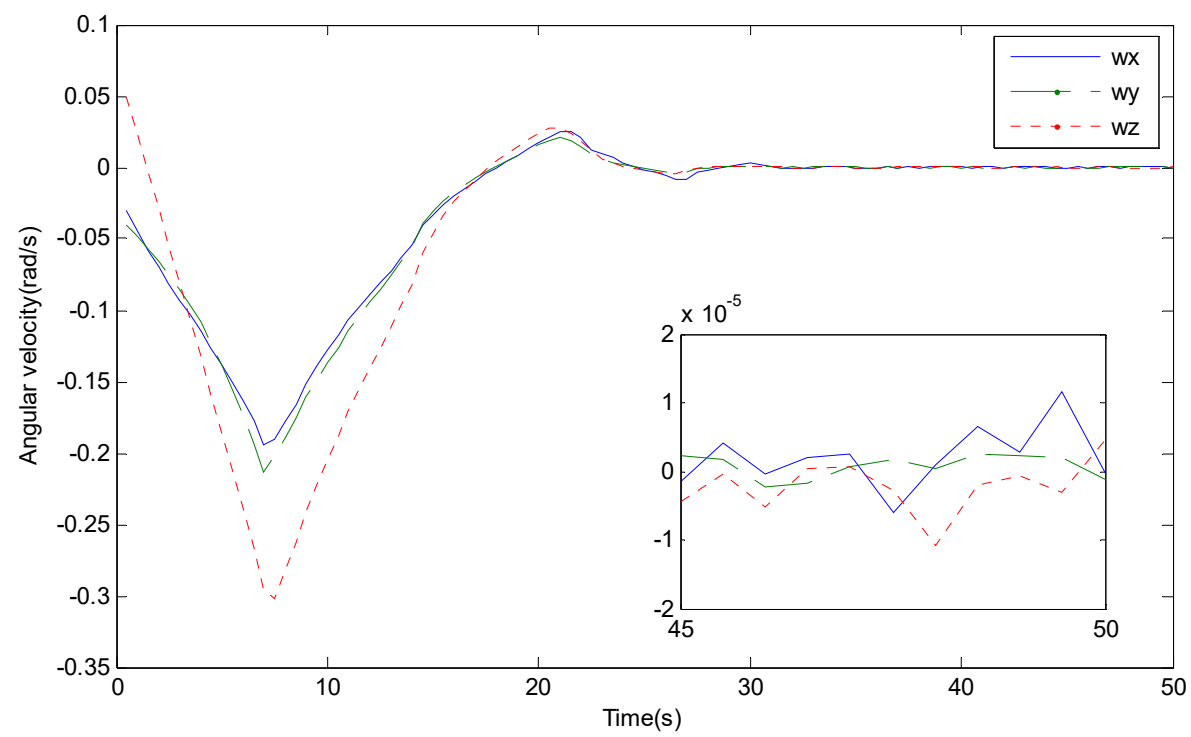

Figure 13. Curve of angular velocity.

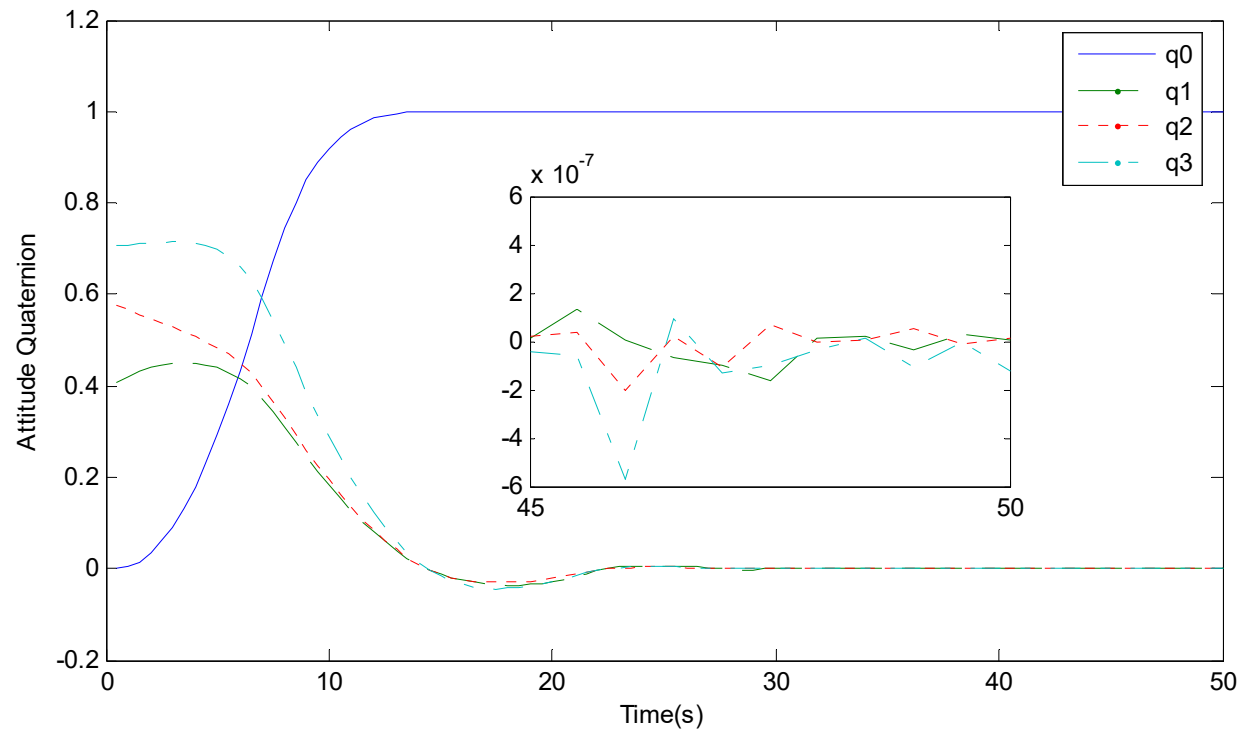

Figure 14. Curve of attitude quaternion. 


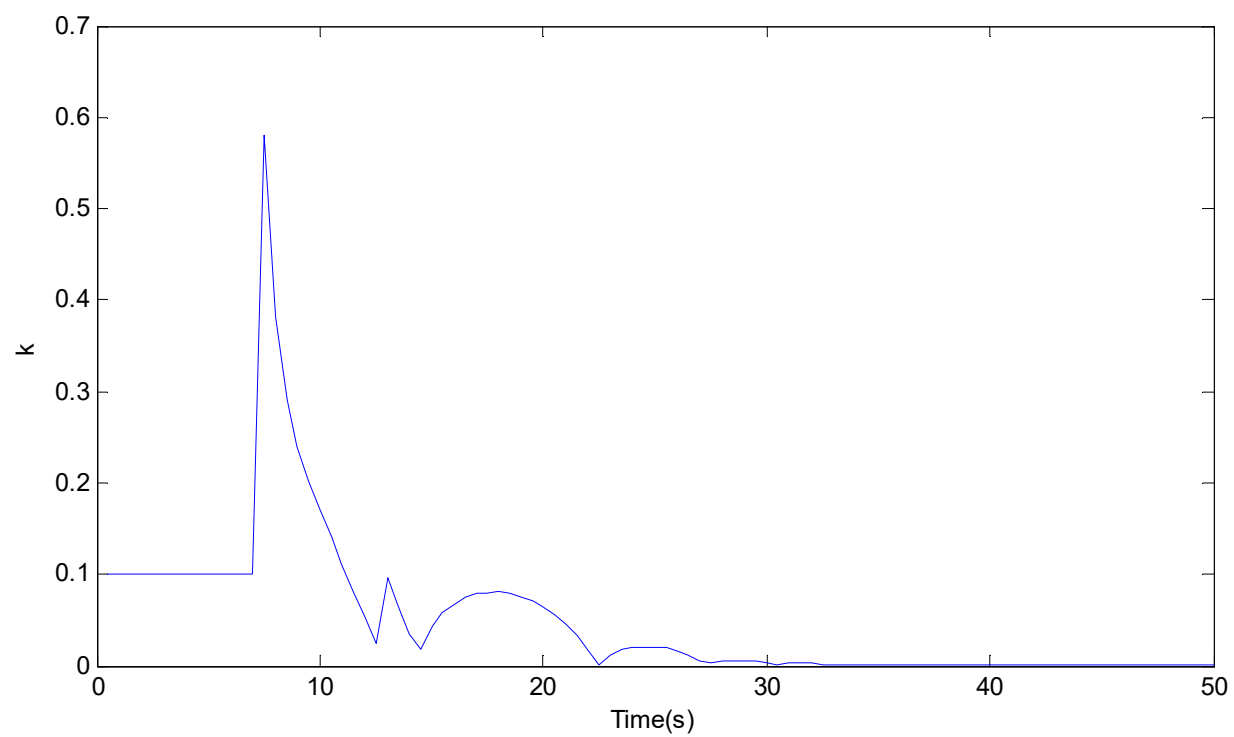

Figure 15. Curve of sliding mode parameter.

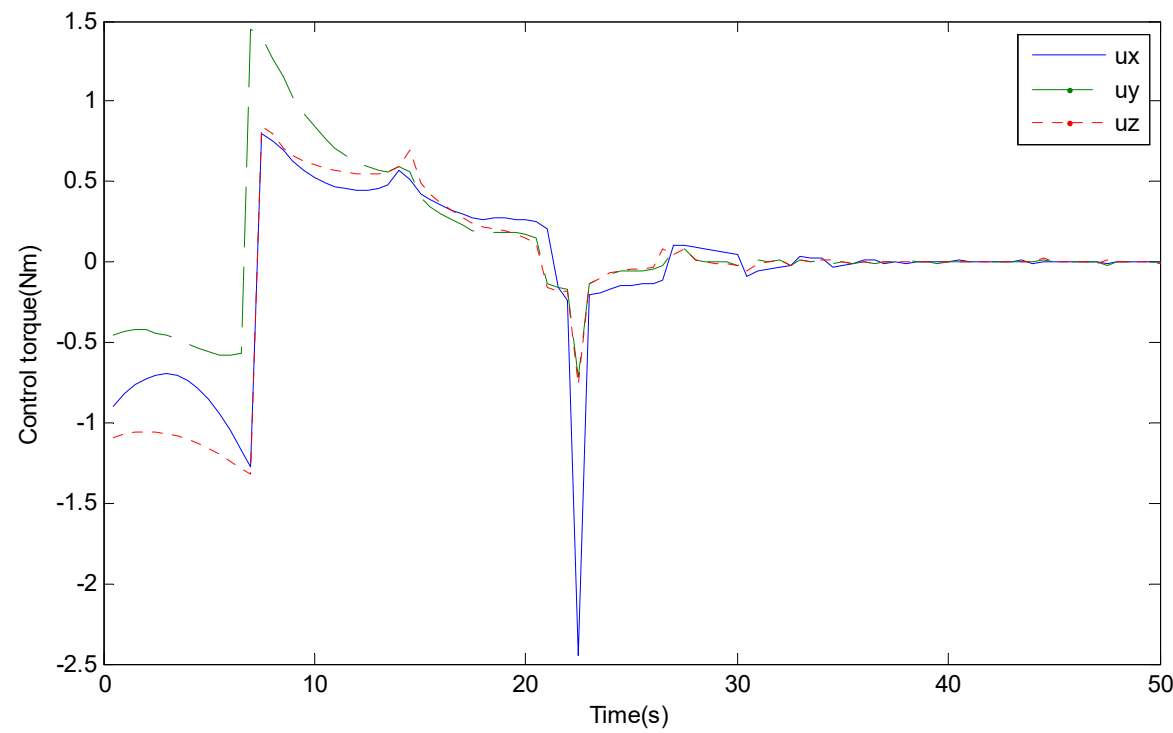

Figure 16. Curve of control torque.

Similar to the discussion in the previous section, in order to demonstrate this property, set system configurations under controller (39) are as follows:

$$
\begin{gathered}
\boldsymbol{d}=1 \times 10^{-2} \times \operatorname{rand}(3 \times 1) \mathrm{Nm}+1 \times 10^{-3} \times\|\boldsymbol{\omega}\| \operatorname{rand}(3 \times 1) \mathrm{Nm} \\
\bar{d}=0, \lambda=0, \boldsymbol{u}^{\prime}=\operatorname{diag}(0.9,0.8,0.7) \boldsymbol{u} \\
\boldsymbol{J}=\operatorname{diag}(30,25,20) \mathrm{kg} \cdot \mathrm{m}^{2}, \hat{\boldsymbol{J}}=\operatorname{diag}(22,18,15) \mathrm{kg} \cdot \mathrm{m}^{2}
\end{gathered}
$$

It could be found that under this configuration system has three aspect perturbations: (1) larger disturbance and no offset term in controller; (2) larger model uncertainty and no offset term in the controller; and (3) control output error in the actuator. The simulation results under strong perturbations are given as follows.

Based on Figures 17-20, it could be found that the system could still converge to the equilibrium point under such perturbations. Comparing this group of simulations with Figures 13-16, it could be found that the main difference is the convergence time. In this group of simulations, the system converges to the equilibrium point about $30 \mathrm{~s}$ 
slower compared with that in the controller (39). Figures 17 and 18 demonstrate that the finite-time sliding mode (31) could achieve a high steady accuracy and resist some typical perturbations such as unknown disturbance, inertia matrix uncertainty and actuator error. This proves that by designing the sliding mode properly, the finite-time stability and strong robustness could both be maintained. Generally, disturbance torque would influence the system steady performance (a larger disturbance would bring a larger sign function term and the system chattering issue would be aggravated), and system steady accuracy would drop.

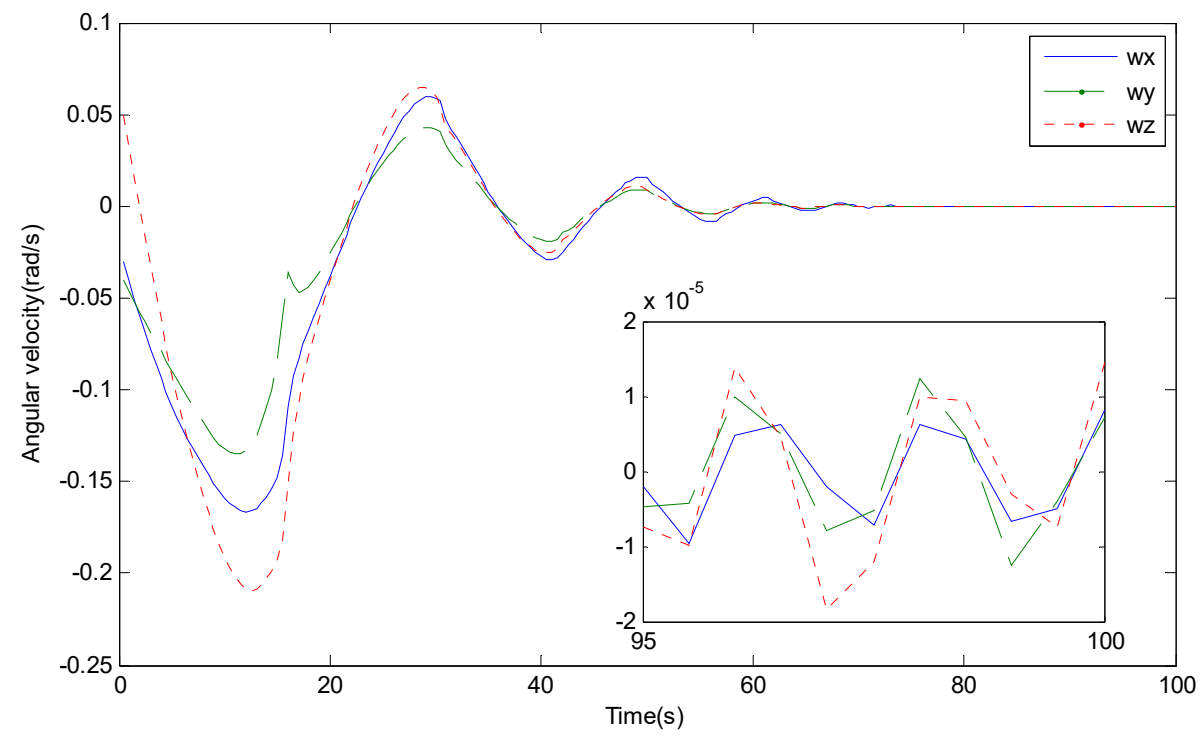

Figure 17. Curve of angular velocity.

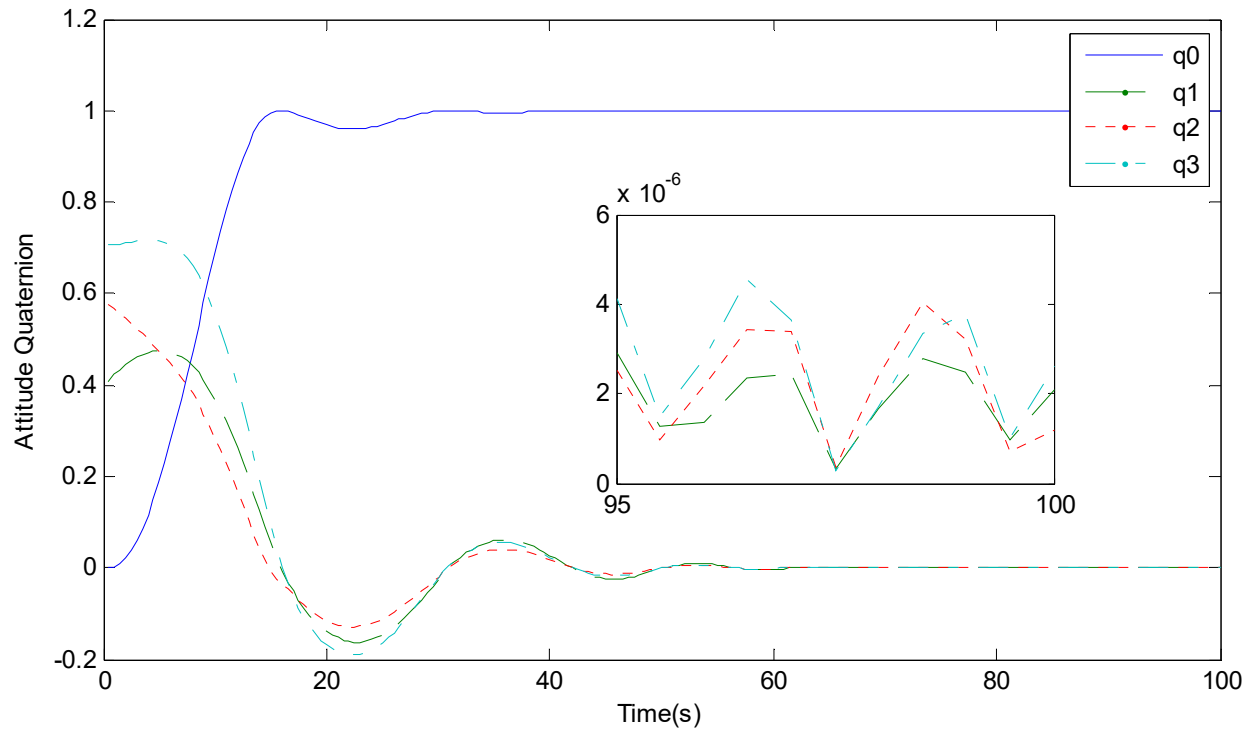

Figure 18. Curve of attitude quaternion. 


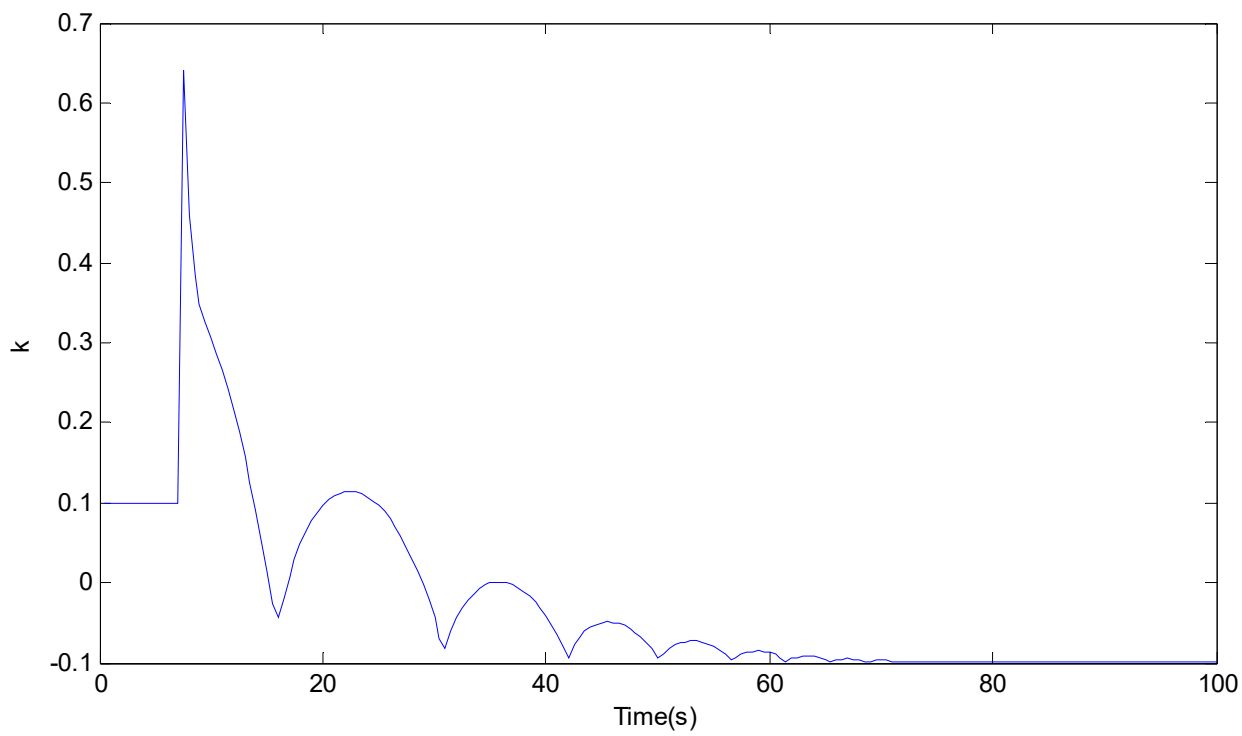

Figure 19. Curve of sliding mode parameter.

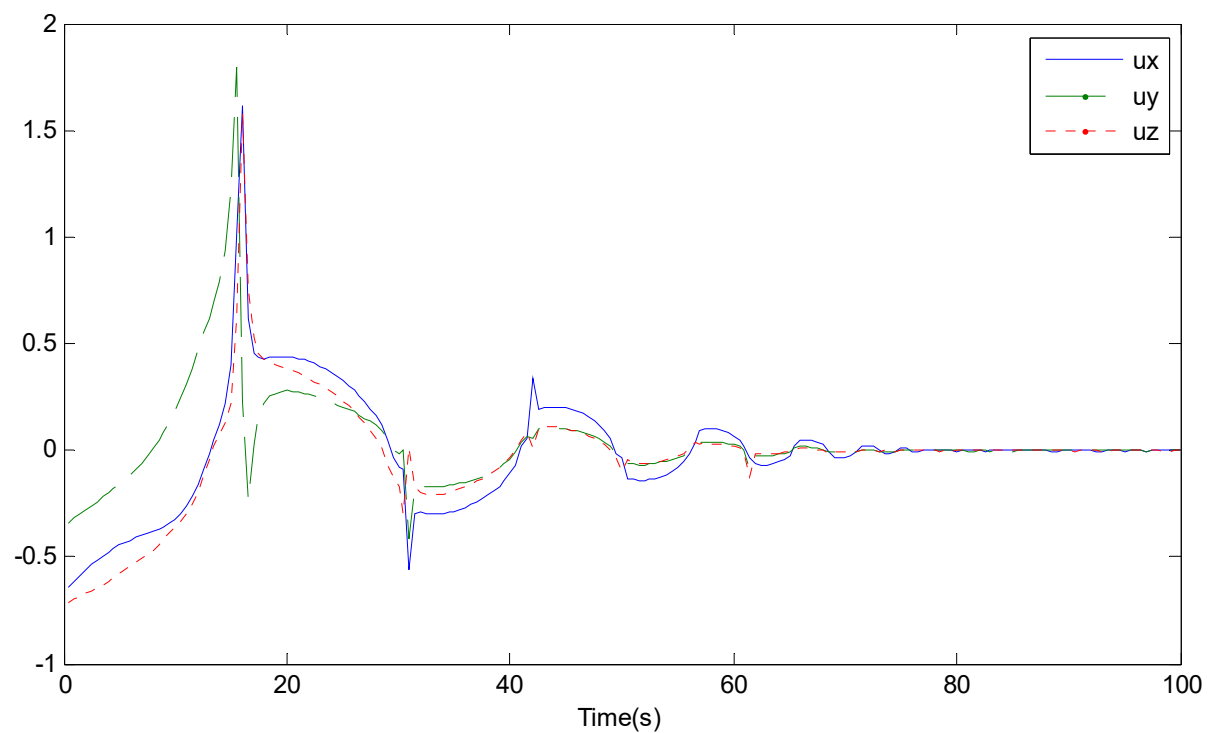

Figure 20. Curve of control torque.

Based on Table 1 it could be found that the proposed controller in this paper mostly maintained the advantage of the standard sliding mode controller, and the system convergence rate is largely improved. Also, the steady accuracy is also largely improved.

Table 1. Simulation results comparison.

\begin{tabular}{cccc}
\hline & Standard Sliding Mode & $\begin{array}{c}\text { Finite Time Based on } \\
\text { Attitude Quaternion }\end{array}$ & $\begin{array}{c}\text { Finite Time Based on } \\
\text { Euler Axis }\end{array}$ \\
\hline Convergence time & Low & Medium & High \\
\hline Steady accuracy & Low & Medium & High \\
\hline Robustness & Strong & Medium & Medium \\
\hline Singularity Issue & None & None & Weak \\
\hline $\begin{array}{c}\text { Controller } \\
\text { Structure }\end{array}$ & Simple & Complex & Complex \\
\hline
\end{tabular}


In this section, performance of the finite-time controller proposed in Section 5 is demonstrated. Compared with the controller in Section 4, the controller (39) could maintain the strong inherent robustness of the standard sliding mode and finite-time stability and the singularity issue of the sliding mode parameter is solved in this situation. The fixed norm of the Euler axis beings this property and this also brings some by-effect to the system, in fact, the control issue is transformed to an attitude determination issue since the small error of the Euler axis could bring large perturbation to system near the system equilibrium point.

\section{Conclusions}

In this paper, finite-time sliding modes are proposed based on standard sliding ones. A novel structure of finite time sliding mode surface is proposed based on a standard sliding mode surface. A system finite time stability is achieved by implementing the update law of sliding mode parameters and the singularity issue is avoided by using the property when angular velocity is reversed to the attitude quaternion.

Generally, by enlarging the sliding mode parameter, the system convergence rate could be improved significantly, and when the update law is designed properly, the desired system performance could be achieved. Also, it could be found that when using attitude quaternion to design the finite-time sliding mode, the sliding mode parameter tends to infinity as the system state converges to zero and the singularity issue of control torque is transformed to the singularity issue of the sliding mode parameter. In order to avoid the breakdown of the computer system caused by this singularity issue, it is necessary to stop updating the parameter when approaching the system equilibrium point. In order to get the strictly finite-time stability, the Euler axis parameter could be used to design a finite time sliding mode. The norm of the Euler axis is fixed, and this property brings some convenience to the design of the finite-time sliding mode. The singularity issue is solved and the system state, control torque and sliding mode parameters are all norm upper bounded. However, the control issue is transformed to a measurement issue since a small error could bring a large perturbation under some typical conditions.

Above all, two kinds of finite time sliding modes are proposed in this paper and each has its advantages and disadvantages. When selecting the sliding mode properly based on the onboard condition and space mission requirement, desired system performance can be achieved.

Author Contributions: Conceptualization, Y.L. and H.L.; methodology, formal analysis and software Y.L.; investigation, H.L.; writing—original draft preparation, Y.L.; writing—review and editing, Y.L. and H.L. All authors have read and agreed to the published version of the manuscript.

Funding: This work was supported partially by the National Natural Science Foundation of China (Project Nos. 61903289, 62003375 and 62103452). The authors greatly appreciate the above financial support. The authors would also like to thank the associate editor and reviewers for their valuable comments and constructive suggestions that helped to improve the paper significantly.

Institutional Review Board Statement: This paper does not have any issues involved with human or animal research.

Informed Consent Statement: This paper does not have any issues involved with human or animal research.

Data Availability Statement: If anyone wants to get the original data of this paper, please contact with You Li by liyou@xidian.edu.cn.

Acknowledgments: This work was supported by National Science Foundation of China (Project Nos.61903289 62003375 and 62103452) and the authors greatly appreciate the above financial support. The authors greatly appreciate the above financial support. The authors would also like to thank the associate editor and reviewers for their valuable comments and constructive suggestions that helped to improve the paper significantly.

Conflicts of Interest: We declare that we have no financial and personal relationships with other people or organizations that can inappropriately influence our work, there is no professional or 
other personal interest of any nature or kind in any product, service and/or company that could be construed as influencing the position presented in, or the review of, the manuscript entitled.

\section{References}

1. Gao, S.; Li, Y.; Yao, S.Y. Robust PD plus Control Algorithm for Satellite Attitude Tracking for Dynamic Targets. Math. Probl. Eng. 2021, 6680994. [CrossRef]

2. Zhang, T.; Zhang, A. Robust Finite-Time Tracking Control for Robotic Manipulators with Time Delay Estimation. Mathematics 2020, 8, 165. [CrossRef]

3. Song, C.; Fei, S.; Cao, J.; Huang, C. Robust Synchronization of Fractional-Order Uncertain Chaotic Systems Based on Output Feedback Sliding Mode Control. Mathematics 2019, 7, 599. [CrossRef]

4. Li, Y.; Ye, D.; Sun, Z.W. Robust finite time control algorithm for satellite attitude control. Aerosp. Sci. Technol. 2017, 68, 46-57. [CrossRef]

5. Li, Y.; Ye, D. Time efficient sliding mode controller based on Bang-Bang logic for satellite attitude control. Aerosp. Sci. Technol. 2018, 75, 342-352. [CrossRef]

6. Li, Y.; Ye, D. Near time-optimal controller based on analytical trajectory planning algorithm for satellite attitude maneuver. Aerosp Sci. Technol. 2019, 84, 497-509.

7. Ye, D.; Li, Y.; Xiao, B.; Li, W.E. Robust finite-time adaptive control algorithm for satellite fast attitude maneuver. J. Frankl. Inst. -Eng. Appl. Math. 2020, 357, 11558-11583. [CrossRef]

8. Zhang, Y.; Nie, Y.; Chen, L. Adaptive Fuzzy Fault-Tolerant Control against Time-Varying Faults via a New Sliding Mode Observer Method. Symmetry 2021, 13, 1615. [CrossRef]

9. Verbin, D.; Lappas, V.J.; Joseph, Z.B. Time efficient angular steering laws for rigid satellites. J. Guid. Control. Dyn. 2011, 34, 878-892. [CrossRef]

10. Rojsiraphisal, T.; Mobayen, S.; Asad, J.H.; Vu, M.T.; Chang, A.; Puangmalai, J. Fast Terminal Sliding Control of Underactuated Robotic Systems Based on Disturbance Observer with Experimental Validation. Mathematics 2021, 9, 1935. [CrossRef]

11. Ye, D.; Zhang, H.Z.; Tian, Y.X.; Zhao, Y.; Sun, Z.W. Fuzzy Sliding Mode Control of Nonparallel-ground-track Imaging Satellite with High Precision. Int. J. Control. Autom. Syst. 2020, 18, 1617-1628. [CrossRef]

12. Ye, D.; Zou, A.M.; Sun, Z.W. Predefined-Time Predefined-Bounded Attitude Tracking Control for Rigid Spacecraft. IEEE Trans. Aerosp. Electron. Syst. 2021. [CrossRef]

13. Wu, S.N.; Radice, G.; Gao, Y.S.; Sun, Z.W. Quaternion-based finite time control for spacecraft attitude tracking. Acta Astronaut. 2011, 69, 48-58. [CrossRef]

14. Wu, S.N.; Radice, G.; Gao, Y.S.; Sun, Z.W. Robust finite-time control for flexible spacecraft attitude maneuver. J. Aerosp. Eng. 2014, 27, 185-190. [CrossRef]

15. Liang, H.Z.; Sun, Z.W.; Wang, J.Y. Finite-time attitude synchronization controllers design for spacecraft formations via behaviorbased approach. Part G J. Aerosp. Eng. 2013, 227, 1737-1753. [CrossRef]

16. Wang, J.Y.; Liang, H.Z.; Sun, Z.W. Dual-quaternion-based finite-time control for spacecraft tracking in six degrees of freedom. Part G J. Aerosp. Eng. 2013, 227, 528-545. [CrossRef]

17. Wang, J.Y.; Liang, H.Z.; Sun, Z.W.; Zhang, S.J.; Liu, M. Finite-time control for spacecraft formation with dual-number-based description. J. Guid. Control. Dyn. 2012, 35, 950-962. [CrossRef]

18. Nguyen, N.P.; Mung, N.X.; Thanh Ha, L.N.N.; Huynh, T.T.; Hong, S.K. Finite-Time Attitude Fault Tolerant Control of Quadcopter System via Neural Networks. Mathematics 2020, 8, 1541. [CrossRef]

19. Khelil, N.; Otis, M.J.D. Finite-Time Stabilization of Homogeneous Non-Lipschitz Systems. Mathematics 2016, 4, 58. [CrossRef]

20. Guo, J.G.; Li, Y.F.; Zhou, J. A new continuous adaptive finite time guidance law against highly maneuvering targets. Aerosp. Sci. Technol. 2019, 85, 40-47. [CrossRef]

21. Xiao, B.; Yin, S.; Wu, L.G. A structure simple controller for satellite attitude tracking maneuver. IEEE Trans. Ind. Electron. 2017, 64, 1436-1446. [CrossRef]

22. Xiao, B.; Yin, S.; Gao, H.J. Tracking control of robotic manipulators with uncertain kinematics and dynamics. IEEE Trans. Ind. Electron. 2016, 63, 6439-6449. [CrossRef]

23. Xiao, B.; Yin, S. Velocity-free Fault tolerant and uncertainty attenuation control for a class of nonlinear systems. IEEE Trans. Ind. Electron. 2016, 63, 4400-4411. [CrossRef]

24. Hu, Q.L.; Zhang, X.X.; Niu, G.L. Observer-based fault tolerant control and experimental verification for rigid spacecraft. Aerosp. Sci. Technol. 2019, 92, 373-386. [CrossRef]

25. Hu, Q.L.; Xie, J.J.; Wang, C.L. Trajectory optimization for accompanying satellite obstacle avoidance. Aerosp. Sci. Technol. 2018, 82-83, 220-233. [CrossRef]

26. Hu, Q.L.; Zhang, J.; Zhang, Y.M. Velocity-free attitude coordinated tracking control for spacecraft formation flying. ISA Trans. 2018, 73, 54-65. [CrossRef] [PubMed]

27. Qiao, J.Z.; Zhang, D.F.; Zhu, Y.K.; Zhang, P.X. Disturbance observer-based finite-time attitude maneuver control for micro satellite under actuator deviation fault. Aerosp. Sci. Technol. 2018, 82-83, 262-271. [CrossRef]

28. Gui, H.C.; Geogre, V. Distributed almost global finite-time attitude consensus of multiple spacecraft without velocity measurements. Aerosp. Sci. Technol. 2018, 75, 284-296. [CrossRef] 
29. Wang, Y.; Ji, H.B. Integrated relative position and attitude control for spacecraft rendezvous with ISS and finite-time convergence. Aerosp. Sci. Technol. 2019, 85, 234-245. [CrossRef]

30. Ai, X.L.; Yu, J.Q. Fixed-time trajectory tracking for a quadrotor with external disturbances: A flatness-based sliding mode control approach. Aerosp. Sci. Technol. 2019, 89, 58-76. [CrossRef]

31. Roman, R.C.; Precup, R.E.; Petriu, E.M. Hybrid data-driven fuzzy active disturbance rejection control for tower crane systems. Eur. J. Control 2021, 58, 373-387. [CrossRef]

32. Hou, Z.S.; Xiong, S.S. On Model-Free Adaptive Control and Its Stability Analysis. IEEE Trans. Autom. Control. 2019, 64, 4555-4569. [CrossRef]

33. Li, K.L.; Boonto, S.; Nuchkrua, T. On-line Self Tuning of Contouring Control for High Accuracy Robot Manipulators under Various Operations. Int. J. Control. Autom. Syst. 2020, 18, 1818-1828. [CrossRef]

34. Li, K.L.; Nuchkrua, T.; Zhao, H.; Yuan, Y.; Boonto, S. Learning-based Adaptive Robust Control of Manipulated Pneumatic Artificial Muscle Driven by H2-based Metal Hydride. In Proceedings of the 2018 IEEE 14th International Conference on Automation Science and Engineering (CASE), Munich, Germany, 20-24 August 2018; pp. 1284-1289. 